\title{
Intramolecular Photocycloaddition of Dioxenones with Alkynes: Formation of Secondary Photoproducts from Cyclobutene Photoadducts
}

\author{
Jeffrey D. Winkler* and Emily C. McLaughlin \\ Department of Chemistry, University of Pennsylvania, Philadelphia, PA 19104 \\ winkler@sas.upenn.edu
}

Table of Contents:

Experimental Section

${ }^{1} \mathrm{H}$ NMR Spectrum of 6

${ }^{13} \mathrm{C}$ NMR Spectrum of 6

IR Spectrum of 6

S12

${ }^{1} \mathrm{H}$ NMR Spectrum of 7

S13

${ }^{13} \mathrm{C}$ NMR Spectrum of 7

S14

IR Spectrum of 7

S 15

${ }^{1} \mathrm{H}$ NMR Spectrum of 8

S16

${ }^{13} \mathrm{C}$ NMR Spectrum of 8

S17

IR Spectrum of 8

S18

${ }^{1} \mathrm{H}$ NMR Spectrum of 11

S19

${ }^{13} \mathrm{C}$ NMR Spectrum of 11

S20 
$\begin{array}{ll}\text { IR Spectrum of } 11 & \text { S21 }\end{array}$

$\begin{array}{ll}{ }^{1} \mathrm{H} \text { NMR Spectrum of } 12 & \text { S22 }\end{array}$

${ }^{13}$ C NMR Spectrum of $12 \quad$ S23

$\begin{array}{lr}\text { IR Spectrum of } 12 & \text { S24 }\end{array}$

$\begin{array}{ll}{ }^{1} \mathrm{H} \text { NMR Spectrum of } 13 & \text { S25 }\end{array}$

${ }^{13}$ C NMR Spectrum of $13 \quad$ S26

$\begin{array}{ll}\text { IR Spectrum of } 13 & \text { S27 }\end{array}$

$\begin{array}{lr}{ }^{1} \mathrm{H} \text { NMR Spectrum of } 17 & \text { S28 }\end{array}$

${ }^{13} \mathrm{C}$ NMR Spectrum of $17 \quad$ S29

$\begin{array}{lr}\text { IR Spectrum of } 17 & \text { S30 }\end{array}$

$\begin{array}{ll}{ }^{1} \mathrm{H} \text { NMR Spectrum of } 18 & \text { S31 }\end{array}$

${ }^{13}$ C NMR Spectrum of $18 \quad$ S32

$\begin{array}{ll}\text { IR Spectrum of } 18 & \text { S33 }\end{array}$

$\begin{array}{lr}\text { X-Ray Structure Determination of } 7 & \text { S34-S40 }\end{array}$ 


\title{
Supporting Information
}

\section{Intramolecular Photocycloaddition of Dioxenones with Alkynes: Formation of Secondary Photoproducts from Cyclobutene Photoadducts}

\author{
Jeffrey D. Winkler* and Emily C. McLaughlin \\ Department of Chemistry, The University of Pennsylvania, Philadelphia, PA 19104
}

\section{Experimental Section}

General Methods. Solvents used for extraction and purification were HPLC grade from Fisher. Unless otherwise indicated, all reactions were run under an inert atmosphere of argon. Anhydrous tetrahydrofuran was obtained via passage through an activated alumina column. ${ }^{1}$ Acetonitrile used for the photoreactions was passed through an alumina column, then irradiated over 3 hours, and redistilled before use. Photochemical reactions were irradiated with a Hanovia medium pressure mercury lamp (450 Watts) through a Pyrex $^{\mathrm{TM}}$ filter. Commercial reagents were used as received. Deuterated solvents were obtained from Cambridge Isotope Labs. Whatman precoated silica gel plates $(250 \mu \mathrm{m})$ were used for analytical TLC. Spots were visualized using $254 \mathrm{~nm}$ ultraviolet light, with either potassium permanganate or anisaldehyde stains as visualizing agents.

Chromatographic purifications were performed on Sorbent Technologies standard silica gel (particle size 32-63 microns). ${ }^{1} \mathrm{H}$ and ${ }^{13} \mathrm{C}$ NMR spectra were recorded on a Bruker AM-500 or DRX-500 spectrometer. Chemical shifts are reported relative to internal chloroform ( $\delta 7.26$ for ${ }^{1} \mathrm{H}, \delta 77.0$ for ${ }^{13} \mathrm{C}$ ). Infrared spectra were recorded on a $\mathrm{KBr}$ plate using a Perkin-Elmer 1600 series Fourier transform infrared spectrometer. High

\footnotetext{
${ }^{1}$ Pangborn, A. B.; Giardello, M. A.; Grubbs, R. H.; Rosen, R. K.; Timmers, F. J. Organometallics 1996, $15,1518$.
} 
resolution mass spectra were obtained by Dr. Rakesh Kohli at the University of Pennsylvania Mass Spectrometry Service Center on an Autospec high resolution doublefocusing electrospray ionization/chemical ionization spectrometer with either DEC 11/73 or OPUS software data system. Single-crystal X-ray diffraction structure determination was performed by Dr. Pat Carroll at the University of Pennsylvania. Melting points were obtained on a Thomas Hoover capillary melting point apparatus and are uncorrected.
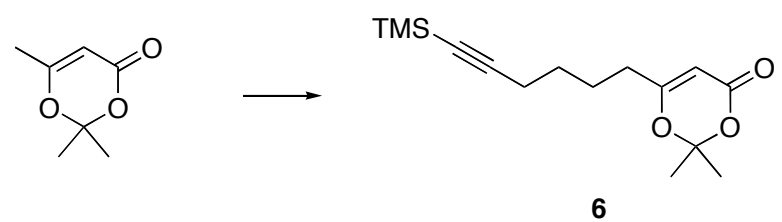

Trimethylsilyl hexynyl dioxenone 6. Lithium diisopropylamide preparation (LDA): $n \operatorname{BuLi}(2.03 \mathrm{~mL}, 4.05 \mathrm{mmol})$ was added dropwise to a solution of diisopropylamine $(0.57$ $\mathrm{mL}, 4.1 \mathrm{mmol})$ in $5.2 \mathrm{~mL}$ THF at $-78^{\circ} \mathrm{C}$. This was stirred for $30 \mathrm{~min}$ at $-78^{\circ} \mathrm{C}$ and HMPA $(0.75 \mathrm{~mL}, 4.3 \mathrm{mmol})$ was added followed by the 2,2,6-trimethyldioxenone $(0.51$ $\mathrm{mL}, 3.9 \mathrm{mmol}$ ) in $2 \mathrm{~mL}$ THF. Stirring continued for $1 \mathrm{hr}$. The 5-trimethylsilyl-4-pentyl iodide $(0.7 \mathrm{~g}, 2.6 \mathrm{mmol})$ in $3.9 \mathrm{~mL}$ THF was then added and the reaction was stirred gradually warming to $25^{\circ} \mathrm{C}$. After 2 hours, the reaction was concentrated and then partitioned between ethyl ether and sat. $\mathrm{NH}_{4} \mathrm{Cl}(30 \mathrm{~mL}$ each). The aqueous layer was acidified with $\mathrm{HCl}$ to $\mathrm{pH} 6$ and then extracted into ether $(2 \times 40 \mathrm{~mL})$, washed with $\mathrm{H}_{2} \mathrm{O}(1$ x $50 \mathrm{~mL})$, sat. $\mathrm{NH}_{4} \mathrm{Cl}(1 \times 50 \mathrm{~mL}), \mathrm{H}_{2} \mathrm{O}(1 \times 50 \mathrm{~mL})$, dried over $\mathrm{MgSO}_{4}$ and concentrated. The residue was purified by silica gel chromatography to yield $0.27 \mathrm{~g} \mathrm{(38 \% )} \mathrm{of} \mathrm{a}$ transparent oil: $\mathrm{R} f 0.25$ (20\% EtOAc/hexanes); ${ }^{1} \mathrm{HNMR}\left(500 \mathrm{MHz}, \mathrm{CDCl}_{3}\right) \delta 5.20$ (s, 1 $\mathrm{H}), 2.21(\mathrm{~m}, 4 \mathrm{H}), 1.64(\mathrm{~s}, 6 \mathrm{H}), 1.63(\mathrm{~m}, 2 \mathrm{H}), 1.52(\mathrm{~m}, 2 \mathrm{H}), 0.13(\mathrm{~s}, 9 \mathrm{H}) ;{ }^{13} \mathrm{CNMR}$ $\left(125 \mathrm{MHz}, \mathrm{CDCl}_{3}\right) \delta 171.43,161.13,106.28,106.19,93.22,85.12,32.98,27.56,24.94$, 24.67, 19.38, 0.02; FTIR (KBr thin film) 2955, 2173, 1743, 1633, 1374, 1271, 1247, $1202,1014,838,761 \mathrm{~cm}^{-1}$. Exact mass calculated for $\mathrm{C}_{15} \mathrm{H}_{24} \mathrm{O}_{3} \mathrm{Si}-\mathrm{CH}_{3}$ (CI) 265.1260, found 265.1249 . 

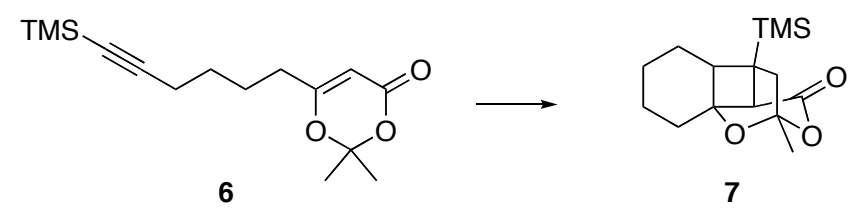

Bridged tetracycle 7. Dioxenone 6 (51 mg, $0.18 \mathrm{mmol}$ ) was dissolved in $54 \mathrm{~mL} \mathrm{CH}_{3} \mathrm{CN}$ and $6 \mathrm{~mL}$ acetone in a Pyrex ${ }^{\mathrm{TM}}$ triple-jacket photo reactor (capacity, $60 \mathrm{~mL}$ ). The solution was degassed under a steady stream of argon over 45 minutes with ice water $\left(\sim 0^{\circ} \mathrm{C}\right)$ running through the condenser. It was then irradiated at $0^{\circ} \mathrm{C}$ over 1.5 hours. The reaction was concentrated to a yellow residue and upon purification by silica gel chromatography yielded $25 \mathrm{mg}$ (49\%) of a yellow solid: m.p. 95-105 ${ }^{\circ} \mathrm{C}, \mathrm{R} f 0.47$ (20\% EtOAc/hexane); ${ }^{1} \mathrm{HNMR}\left(500 \mathrm{MHz}, \mathrm{CDCl}_{3}\right) \delta 3.38(\mathrm{~s}, 1 \mathrm{H}), 2.13(\mathrm{~d}, J=13.3 \mathrm{~Hz}, 1 \mathrm{H})$, $2.05(\mathrm{~d}, J=13.3 \mathrm{~Hz}, 1 \mathrm{H}), 2.00(\mathrm{~m}, 1 \mathrm{H}), 1.85$ (ddd, $J=4.5,4.5,15.8 \mathrm{~Hz}, 1 \mathrm{H}), 1.76$ (m, $1 \mathrm{H}), 1.63(\mathrm{~s}, 3 \mathrm{H}), 1.62(\mathrm{~m}, 1 \mathrm{H}), 1.43$ (dddd, $J=4.9,4.9,15.5,15.2 \mathrm{~Hz}, 2 \mathrm{H}), 1.33$ (m, $2 \mathrm{H}), 1.00(\mathrm{ddd}, J=12.9,12.9,12.9 \mathrm{~Hz}, 1 \mathrm{H}), 0.03(\mathrm{~s}, 9 \mathrm{H}) ;{ }^{13} \mathrm{CNMR}\left(125 \mathrm{MHz}, \mathrm{CDCl}_{3}\right)$ \& 170.78, 102.97, 81.74, 50.97, 42.85, 41.25, 31.02, 30.92, 28.15, 24.20, 22.84, 22.76, -2.29; FTIR (KBr thin film) 2938, 1743, 1287, 1249, $839 \mathrm{~cm}^{-1}$. Exact mass calculated for $\mathrm{C}_{15} \mathrm{H}_{24} \mathrm{O}_{3} \mathrm{Si}+\mathrm{Na}(\mathrm{ES}+)$ 303.1393, found 303.1393 .

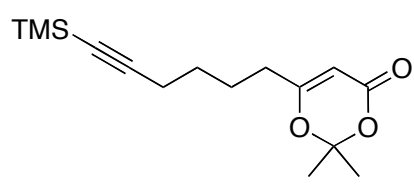

6

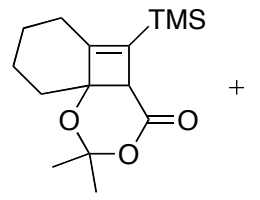

8

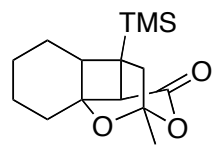

7

The dioxenone 6 ( $90 \mathrm{mg}$; $0.32 \mathrm{mmol}$ ) was dissolved in $54 \mathrm{~mL} \mathrm{CH}_{3} \mathrm{CN}$ and $6 \mathrm{~mL}$ acetone in a triple-jacket, Pyrex ${ }^{\mathrm{TM}}$ photoreactor (capacity, $60 \mathrm{~mL}$ ). The reaction was degassed under a steady stream of argon over 1 hour while ice water $\left(\sim 0^{\circ} \mathrm{C}\right)$ ran through the condenser. Next, the reaction was irradiated for 10 minutes at $0^{\circ} \mathrm{C}$ before it was concentrated to residue. The residue was purified by silica gel chromatography to yield $36 \mathrm{mg}(40 \%)$ of 8 and $16 \mathrm{mg}(18 \%)$ of 7. 
Cyclobutene 8: $\mathrm{R} f 0.6$ (20\% EtOAc/hexane); ${ }^{1} \mathrm{HNMR}\left(500 \mathrm{MHz}, \mathrm{CDCl}_{3}\right) \delta 3.31$ (s, 1 H), $2.33(\mathrm{dd}, J=4.4,13.4 \mathrm{~Hz}, 1 \mathrm{H}), 2.18(\mathrm{~m}, 1 \mathrm{H}), 2.13(\mathrm{~m}, 1 \mathrm{H}), 1.93(\mathrm{~m}, 1 \mathrm{H}), 1.67$ (m, 2 H), 1.59 (s, $3 \mathrm{H}), 1.55$ (s, $3 \mathrm{H}), 1.44$ (ddd, $J=3.2,13.1,13.1 \mathrm{~Hz}, 1 \mathrm{H}), 1.25$ (m, 1 $\mathrm{H}), 0.15(\mathrm{~s}, 9 \mathrm{H}) ;{ }^{13} \mathrm{CNMR}\left(125 \mathrm{MHz}, \mathrm{CDCl}_{3}\right) \delta 171.17,167.95,138.72,106.40,75.63$, 50.53, 37.54, 29.38, 27.75, 27.16, 26.27, 20.99, -1.43; FTIR (KBr thin film) 2945, 1738, $1385,1285,1247,1182,1108,978,842 \mathrm{~cm}^{-1}$. Exact mass calculated for $\mathrm{C}_{15} \mathrm{H}_{24} \mathrm{O}_{3} \mathrm{Si}-\mathrm{Me}$ (CI) 265.1260, found 265.1255.

Bridged tetracycle 7: m.p. $95-105^{\circ} \mathrm{C}, \mathrm{R} f 0.47$ (20\% EtOAc/hexane); ${ }^{1} \mathrm{HNMR}$ (500 $\left.\mathrm{MHz}, \mathrm{CDCl}_{3}\right) \delta 3.38(\mathrm{~s}, 1 \mathrm{H}), 2.13(\mathrm{~d}, J=13.3 \mathrm{~Hz}, 1 \mathrm{H}), 2.05(\mathrm{~d}, J=13.3 \mathrm{~Hz}, 1 \mathrm{H}), 2.00$ (m, $1 \mathrm{H}), 1.85$ (ddd, $J=4.5,4.5,15.8 \mathrm{~Hz}, 1 \mathrm{H}), 1.76(\mathrm{~m}, 1 \mathrm{H}), 1.63(\mathrm{~s}, 3 \mathrm{H}), 1.62(\mathrm{~m}, 1$ H), 1.43 (dddd, $J=4.9,4.9,15.5,15.2 \mathrm{~Hz}, 2 \mathrm{H}), 1.33$ (m, $2 \mathrm{H}$ ), 1.00 (ddd, $J=12.9,12.9$, $12.9 \mathrm{~Hz}, 1 \mathrm{H}), 0.03$ (s, $9 \mathrm{H}) ;{ }^{13} \mathrm{CNMR}\left(125 \mathrm{MHz}, \mathrm{CDCl}_{3}\right.$ ) $\delta$ 170.78, 102.97, 81.74, 50.97, $42.85,41.25,31.02,30.92,28.15,24.20,22.84,22.76,-2.29$; FTIR (KBr thin film) 2938, $1743,1287,1249,839 \mathrm{~cm}^{-1}$. Exact mass calculated for $\mathrm{C}_{15} \mathrm{H}_{24} \mathrm{O}_{3} \mathrm{Si}+\mathrm{Na}(\mathrm{ES}+)$ 303.1393, found 303.1393 .

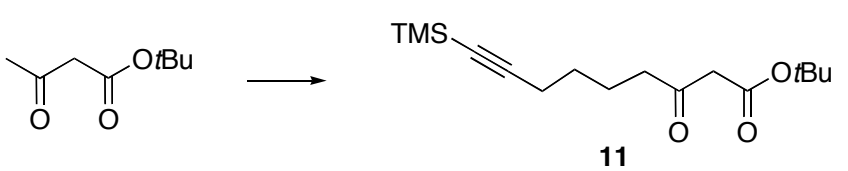

$\beta$-Ketoester 11. The $t$-butyl acetoacetate $(1.65 \mathrm{~g} ; 9.9 \mathrm{mmol})$ was added dropwise to a suspension at $25^{\circ} \mathrm{C}$ of $\mathrm{NaH}(10.3 \mathrm{~g} ; 12.5 \mathrm{mmol})$ in $28 \mathrm{~mL}$ of THF. It was stirred over 30 minutes, then the reaction was cooled to $0^{\circ} \mathrm{C} . n \mathrm{BuLi}(5.3 \mathrm{~mL} ; 12.5 \mathrm{mmol})$ was added and it was stirred an additional $15 \mathrm{~min}$. At $0^{\circ} \mathrm{C} 5$-trimethylsilyl-4-pentyl iodide $(2.21 \mathrm{~g}$; $8.3 \mathrm{mmol}$ ) was added in a solution with $5 \mathrm{~mL}$ THF. The reaction was allowed to gradually warm to $25^{\circ} \mathrm{C}$, and after 4 hours, it was quenched by addition of $20 \mathrm{~mL}$ sat'd $\mathrm{NH}_{4} \mathrm{Cl}$. The mixture was extracted into $\mathrm{Et}_{2} \mathrm{O}(2 \times 40 \mathrm{~mL})$, the organics dried over $\mathrm{MgSO}_{4}$, and concentrated to a yellow oil. The crude oil was purified by silica gel 
chromatography to yield $1.57 \mathrm{~g}(64 \%)$ of a yellow oil: $\mathrm{R} f 0.43$ (20\% EtOAc/hexane); ${ }^{1} \mathrm{HNMR}\left(500 \mathrm{MHz}, \mathrm{CDCl}_{3}\right) \delta 3.32(\mathrm{~s}, 2 \mathrm{H}), 2.54(\mathrm{dd}, J=7.1,7.1 \mathrm{~Hz}, 2 \mathrm{H}), 2.21(\mathrm{dd}, J=$ 7.4, 7.4 Hz, $2 \mathrm{H}$ ), 1.68 (dddd, $J=7.1,7.1,7.1,7.1 \mathrm{~Hz}, 2 \mathrm{H}), 1.51$ (dddd, $J=7.1,7.1,7.1$, $7.1 \mathrm{~Hz}, 2 \mathrm{H}), 1.45$ (s, $9 \mathrm{H}), 0.12$ (s, $9 \mathrm{H}) ;{ }^{13} \mathrm{CNMR}\left(125 \mathrm{MHz}, \mathrm{CDCl}_{3}\right) \delta 202.84,166.38$, 106.74, 84.80, 81.87, 50.57, 42.26, 28.30, 27.95, 22.56, 19.61, 0.10; FTIR (KBr thin film) $2958,2174,1738,1717,1251,1154,843,760 \mathrm{~cm}^{-1}$. Exact mass calculated for $\mathrm{C}_{16} \mathrm{H}_{28} \mathrm{O}_{3} \mathrm{Si}+\mathrm{H}(\mathrm{CI}) 297.1886$ found 297.1910.

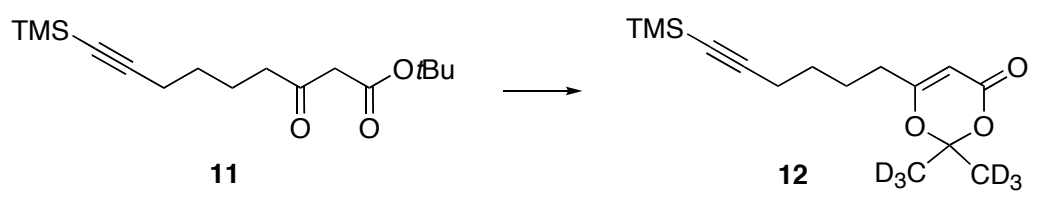

Deuterated trimethylsilyl hexynyl dioxenone 12. The $\beta$-ketoester $11(0.25 \mathrm{~g}, 0.85$ mmol) was dissolved in $4 \mathrm{~mL}$ acetone- $d 6$ and cooled to $-78^{\circ} \mathrm{C}$. Trifluoroacetic anhydride $(0.75 \mathrm{~mL}, 1.14 \mathrm{M})$ was added directly via syringe followed by dropwise addition of trifluoroacetic acid $(3.7 \mathrm{~mL}, 0.23 \mathrm{M})$ and then acetic anhydride $(0.6 \mathrm{~mL}$, $1.47 \mathrm{M})$. The reaction was stirred at $-78^{\circ} \mathrm{C}$ for $30 \mathrm{~min}$, then gradually warmed to $25^{\circ} \mathrm{C}$ and allowed to stir for $12 \mathrm{hr}$. Quenched with $20 \mathrm{~mL}$ sat. $\mathrm{NaHCO}_{3}$ and solid $\mathrm{NaHCO}_{3}$ to reach $\mathrm{pH} 7$, the reaction was extracted into ethyl ether $(3 \times 10 \mathrm{~mL})$, dried over $\mathrm{MgSO}_{4}$, and concentrated. The resulting residue was purified by silica gel chromatography (10\% EtOAc/hexanes) to afford $0.127 \mathrm{~g}(45 \%)$ of a transparent oil: $\operatorname{Rf} 0.17(20 \%$ EtOAc/hexane); ${ }^{1} \mathrm{HNMR}\left(500 \mathrm{MHz}, \mathrm{CDCl}_{3}\right) \delta 5.21$ (s, $\left.1 \mathrm{H}\right), 2.22$ (m, $\left.4 \mathrm{H}\right), 1.64$ (dddd, $J$ = 7.4, 7.4, 7.4, 7.4 Hz, $2 \mathrm{H}), 1.52$ (dddd, $J=7.2,7.2,7.2,7.2 \mathrm{~Hz}, 2 \mathrm{H}), 0.11$ (s, $9 \mathrm{H})$; ${ }^{13} \mathrm{CNMR}\left(125 \mathrm{MHz}, \mathrm{CDCl}_{3}\right) \delta 171.45,161.15,106.29,93.23,85.14,32.99,27.58,24.69$, 24.09 (m), 19.39, 0.03; FTIR (KBr thin film) 2954, 2901, 2173, 1746, 1717, 1634, 1380, $1280,1249,1190,951,840,761 \mathrm{~cm}^{-1}$. Exact mass calculated for $\mathrm{C}_{15} \mathrm{H}_{18} \mathrm{D}_{6} \mathrm{O}_{3} \mathrm{Si}-\mathrm{CH}_{3}$ (CI) 271.1636, found 271.1629. 

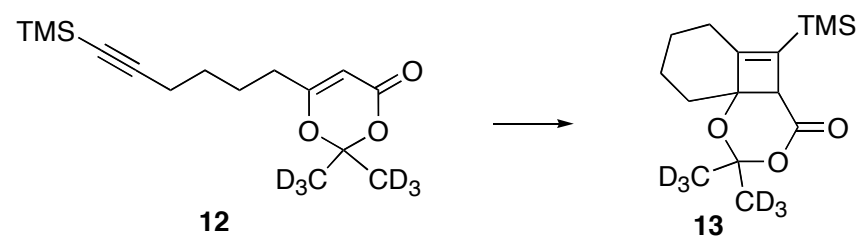

Cyclobutene 13. The dioxenone $12(60 \mathrm{mg} ; 0.21 \mathrm{mmol})$ was dissolved in $54 \mathrm{~mL} \mathrm{CH}_{3} \mathrm{CN}$ and $6 \mathrm{~mL}$ acetone in a triple-jacket, pyrex photoreactor (capacity $=60 \mathrm{~mL}$ ). The reaction was degassed under a steady stream of argon over 1 hour while ice water $\left(\sim 0^{\circ} \mathrm{C}\right)$ ran through the condenser. Next, the reaction was irradiated for 10 minutes at $0^{\circ} \mathrm{C}$ before it was concentrated to residue. The residue was purified by silica gel chromatography to yield $28 \mathrm{mg}$ (47\%) of a yellow oil: Rf 0.5 (20\% EtOAc/hexane); ${ }^{1} \mathrm{HNMR}(500 \mathrm{MHz}$, $\left.\mathrm{CDCl}_{3}\right) \delta 3.31(\mathrm{~d}, J=2.1 \mathrm{~Hz}, 1 \mathrm{H}), 2.34(\mathrm{dd}, J=4.4,13.3 \mathrm{~Hz}, 1 \mathrm{H}), 2.17(\mathrm{~m}, 1 \mathrm{H}), 2.13$ (m, 1 H), 1.94 (m, 1 H), 1.64-1.68 (m, 2 H), 1.44 (ddd, $J=4.3,13.2,13.2 \mathrm{~Hz}, 1 \mathrm{H}), 1.25$ (m, $1 \mathrm{H}), 0.16(\mathrm{~s}, 9 \mathrm{H}) ;{ }^{13} \mathrm{CNMR}\left(125 \mathrm{MHz}, \mathrm{CDCl}_{3}\right) \delta$ 171.20, 167.97, 138.77, 75.66, 50.60, 37.58, 27.20, 26.30, 21.04, -1.40; FTIR ( $\mathrm{KBr}$ thin film) 2944, 1736, 1284, 1253, 999, $841 \mathrm{~cm}^{-1}$. Exact mass calculated for $\mathrm{C}_{15} \mathrm{H}_{18} \mathrm{O}_{3} \mathrm{SiD}_{6}+\mathrm{H}(\mathrm{CI}) 286.1949$, found 286.1950 .

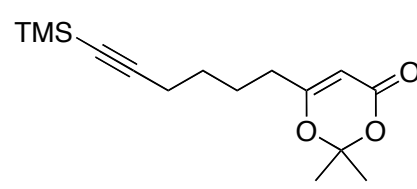

6

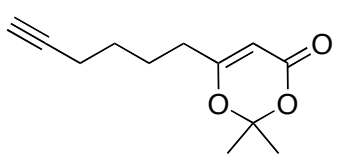

17

Hexynyl dioxenone 17. The protected alkyne $6(160 \mathrm{mg} ; 0.55 \mathrm{mmol})$ was dissolved in 5 $\mathrm{mL}$ THF then cooled to $0^{\circ} \mathrm{C}$. The reaction was treated with TBAF $(0.61 \mathrm{~mL} ; 0.61 \mathrm{mmol})$ and stirred at $0^{\circ} \mathrm{C}$ over $20 \mathrm{~min}$. Next, $5 \mathrm{ml} \mathrm{H}_{2} \mathrm{O}$ was added and the reaction was extracted into $\mathrm{Et}_{2} \mathrm{O}(3 \times 10 \mathrm{~mL})$. The extracts were dried over $\mathrm{NaSO}_{4}$ and concentrated to yield $126 \mathrm{mg}$ (quantitative yield) of a yellow oil: $\mathrm{R} f 0.29$ (20\% EtOAc/hexanes); ${ }^{1} \mathrm{HNMR}$ $\left(500 \mathrm{MHz}, \mathrm{CDCl}_{3}\right) \delta 5.19$ (s, $\left.1 \mathrm{H}\right), 2.19(\mathrm{~m}, 4 \mathrm{H}), 1.92$ (d, J = 2.7 Hz, $\left.1 \mathrm{H}\right), 1.64$ (bs, 8 $\mathrm{H}), 1.53(\mathrm{dd}, J=6.1,6.1 \mathrm{~Hz}, 2 \mathrm{H}) ;{ }^{13} \mathrm{CNMR}\left(125 \mathrm{MHz}, \mathrm{CDCl}_{3}\right) \delta 171.35,161.09$, 
106.20, 93.19, 83.46, 68.76, 32.93, 27.47, 24.90, 24.58, 17.94; FTIR ( $\mathrm{KBr}$ thin film) $3292,2942,1727,1632,1391,1273,1204,1014,901 \mathrm{~cm}^{-1}$. Exact mass calculated for $\mathrm{C}_{12} \mathrm{H}_{16} \mathrm{O}_{3}+\mathrm{H}(\mathrm{CI})$ 209.1177, found 209.1124.

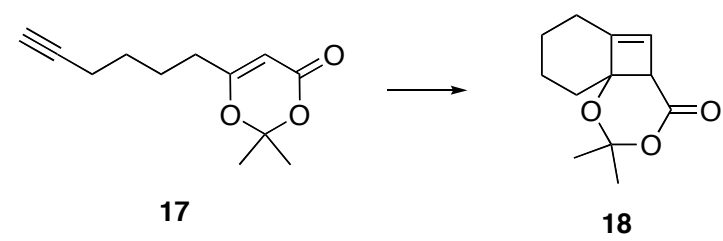

Cyclobutene 18. Dioxenone 17 (40 mg; $0.19 \mathrm{mmol}$ ) was dissolved in $54 \mathrm{~mL} \mathrm{CH}_{3} \mathrm{CN}$ and $6 \mathrm{~mL}$ acetone in a triple-jacket Pyrex ${ }^{\mathrm{TM}}$ photoreactor (capacity $=60 \mathrm{~mL}$ ). The reaction was degassed under a steady stream of argon over 1 hour while ice water $\left(\sim 0^{\circ} \mathrm{C}\right)$ ran through the condenser. Next, the reaction was irradiated for $10 \mathrm{~min}$ before it was concentrated to residue. The residue was purified by silica gel chromatography to yield $30 \mathrm{mg}(75 \%)$ of a transparent residue: $\mathrm{R} f 0.32$ (20\% EtOAc/hexane); ${ }^{1} \mathrm{HNMR}$ (500 $\left.\mathrm{MHz}, \mathrm{CDCl}_{3}\right) \delta 5.73(\mathrm{~s}, 1 \mathrm{H}), 3.37$ (s, $\left.1 \mathrm{H}\right), 2.26(\mathrm{dd}, J=4.6,13.6 \mathrm{~Hz}, 1 \mathrm{H}), 2.21(\mathrm{dd}, J=$ 0.0, 13.6 Hz, $1 \mathrm{H}), 2.09$ (m, $1 \mathrm{H}), 1.91(\mathrm{~m}, 1 \mathrm{H}), 1.66(\mathrm{~m}, 2 \mathrm{H}), 1.59$ (s, $3 \mathrm{H}), 1.57$ (s, 3 H), 1.47 (ddd, $J=4.4,13.1,13.1 \mathrm{~Hz}, 1 \mathrm{H}$ ), 1.27 (dddd, $J=4.7,12.6,12.6,17.0 \mathrm{~Hz}, 1$ $\mathrm{H}) ;{ }^{13} \mathrm{CNMR}\left(125 \mathrm{MHz}, \mathrm{CDCl}_{3}\right) \delta$ 171.08, 157.08, 121.80, 106.89, 76.04, 48.62, 36.73, 28.96, 27.88, 26.40, 24.95, 20.77; FTIR (KBr thin film) 2939, 2857, 1737, 1386, 1290, $1115,1030 \mathrm{~cm}^{-1}$. Exact mass calculated for $\mathrm{C}_{12} \mathrm{H}_{16} \mathrm{O}_{3}+\mathrm{Na}(\mathrm{ES}+)$ 231.0997, found 231.0987. 


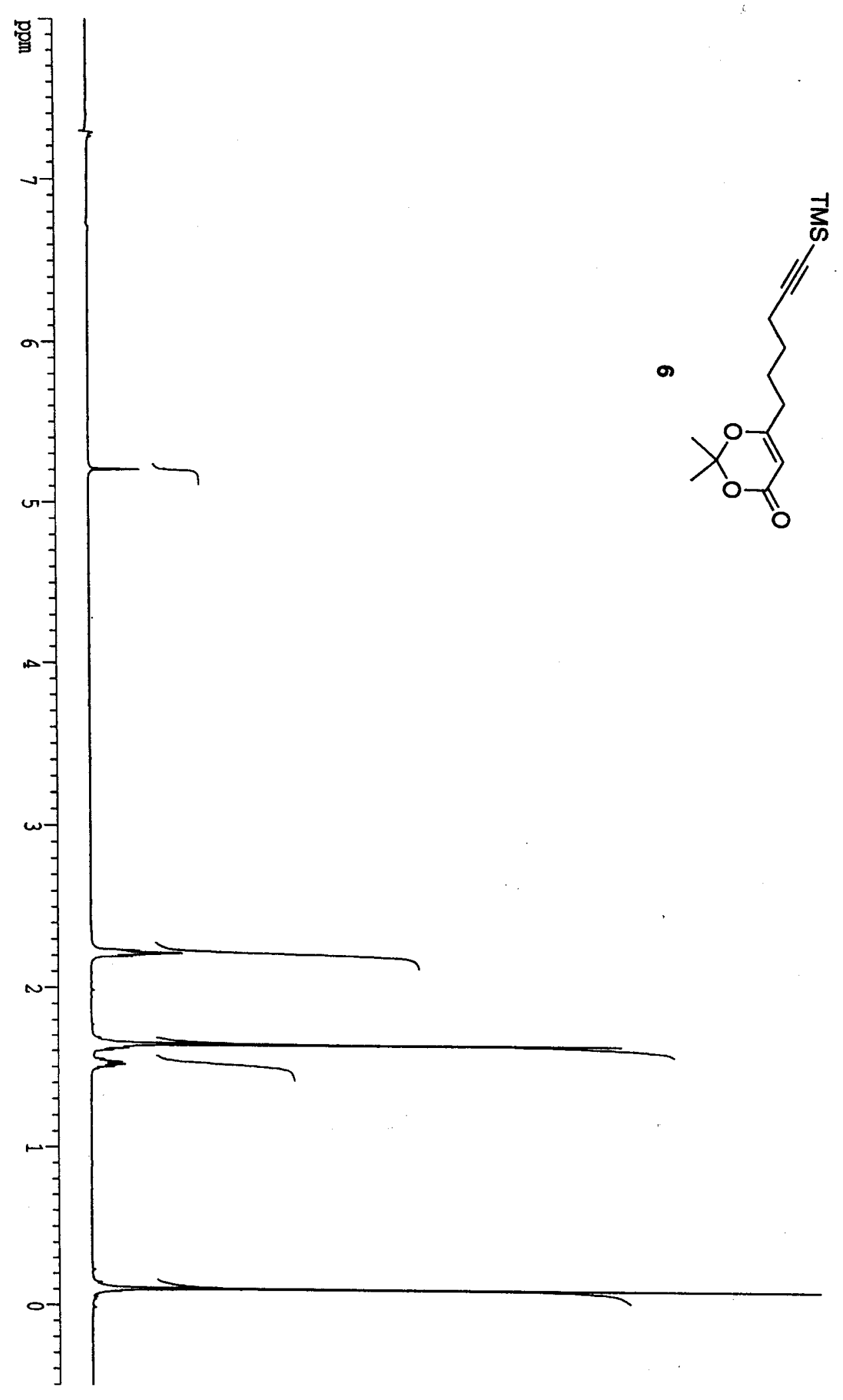




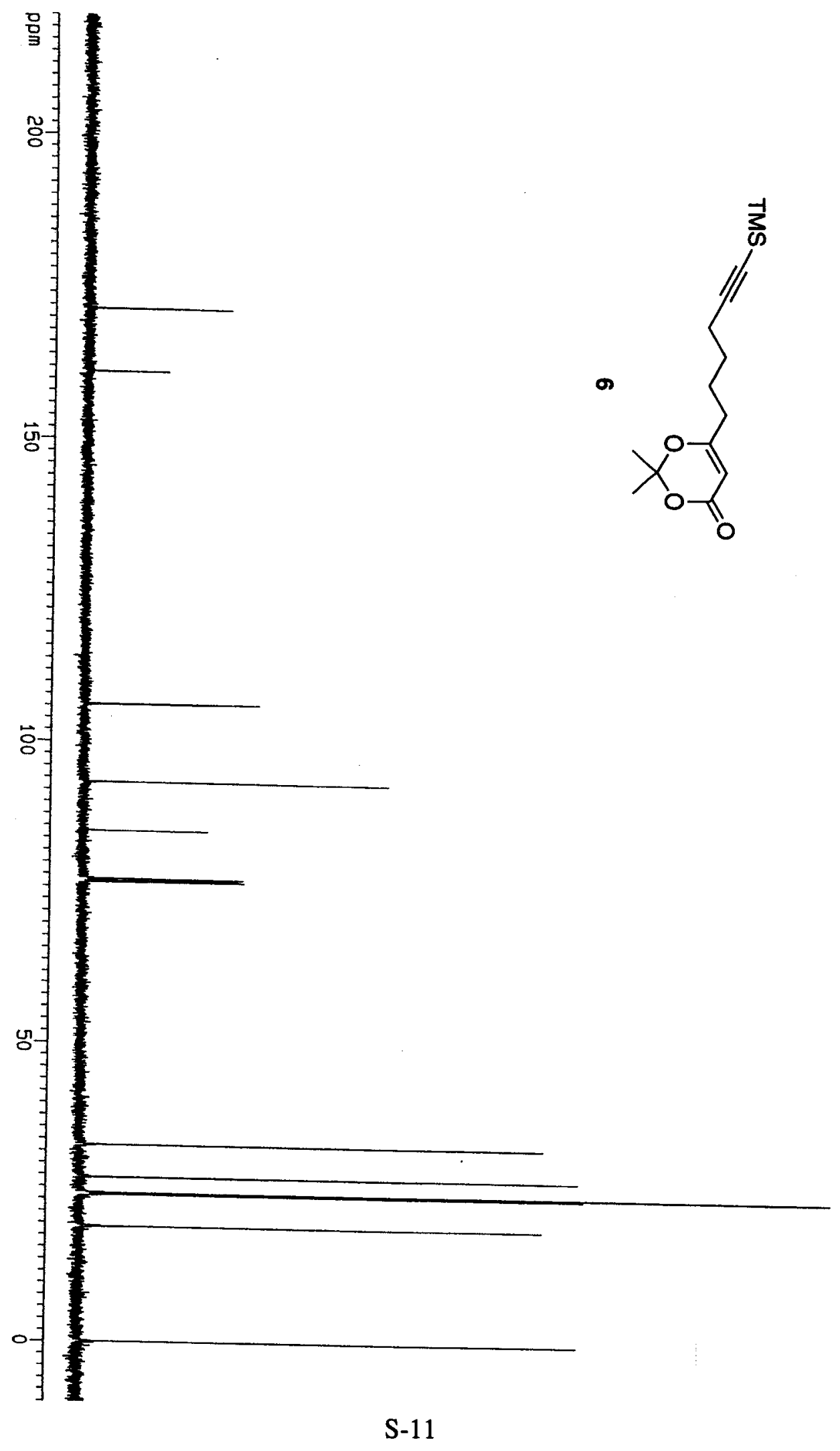




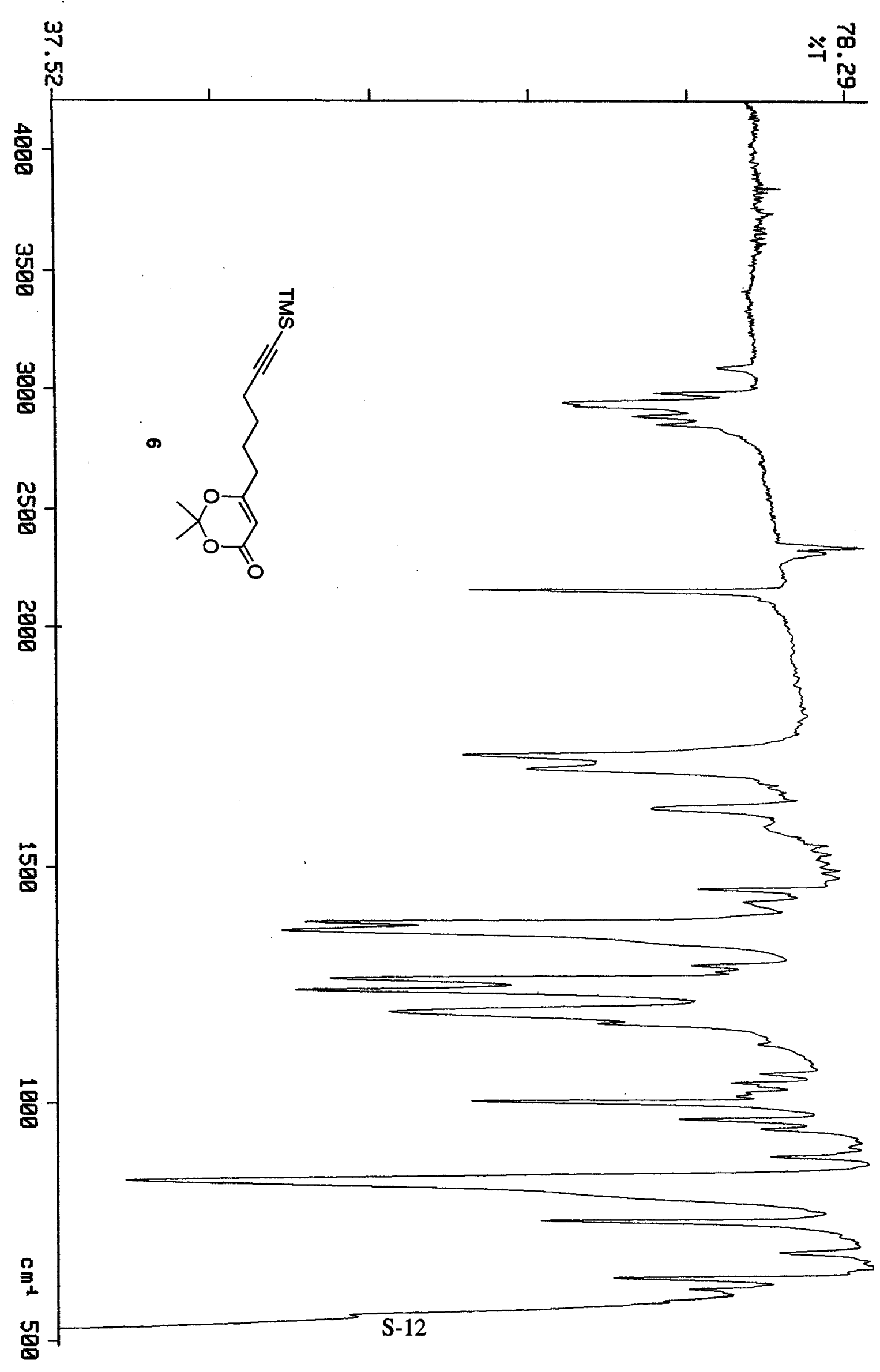




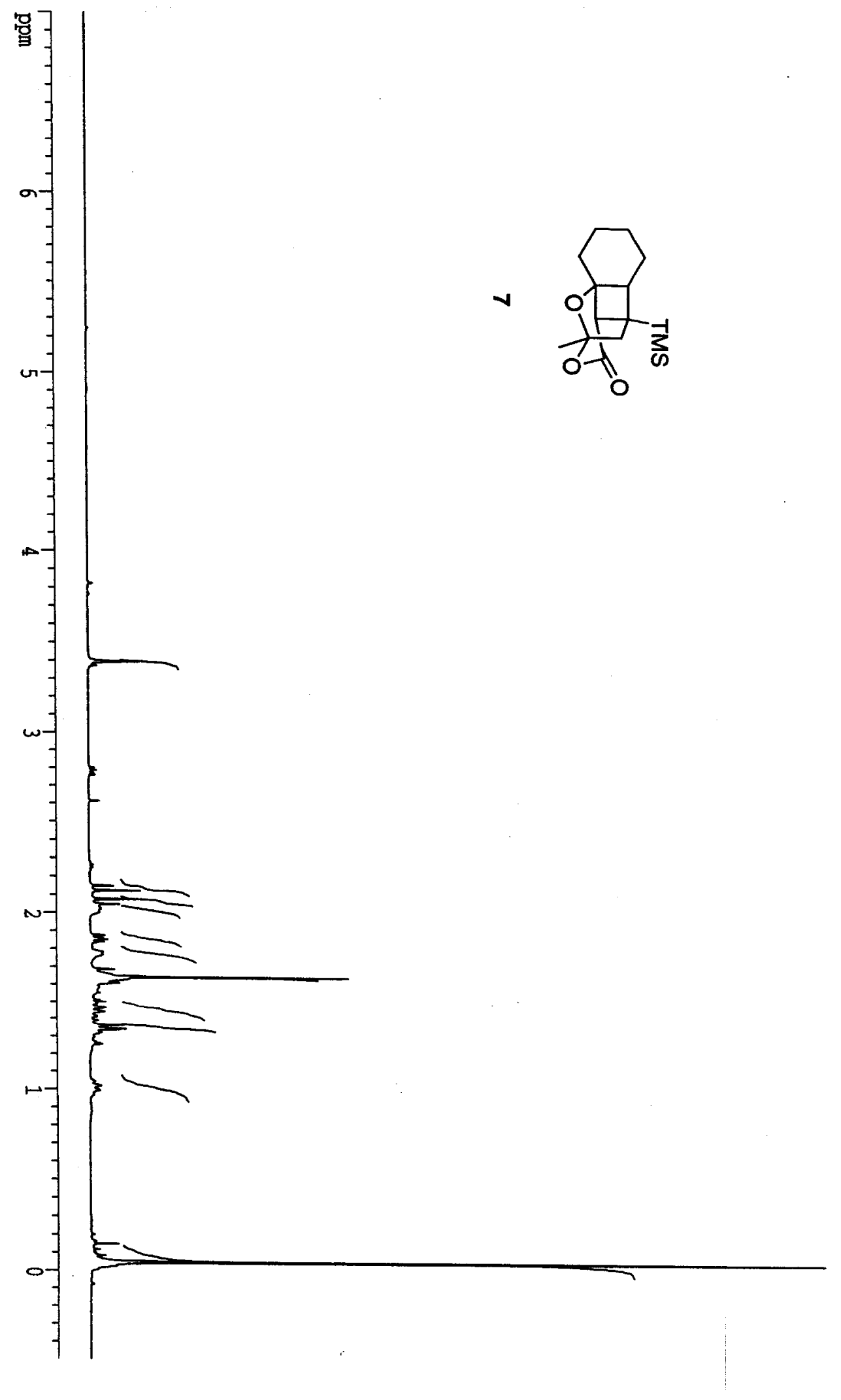

S-13 

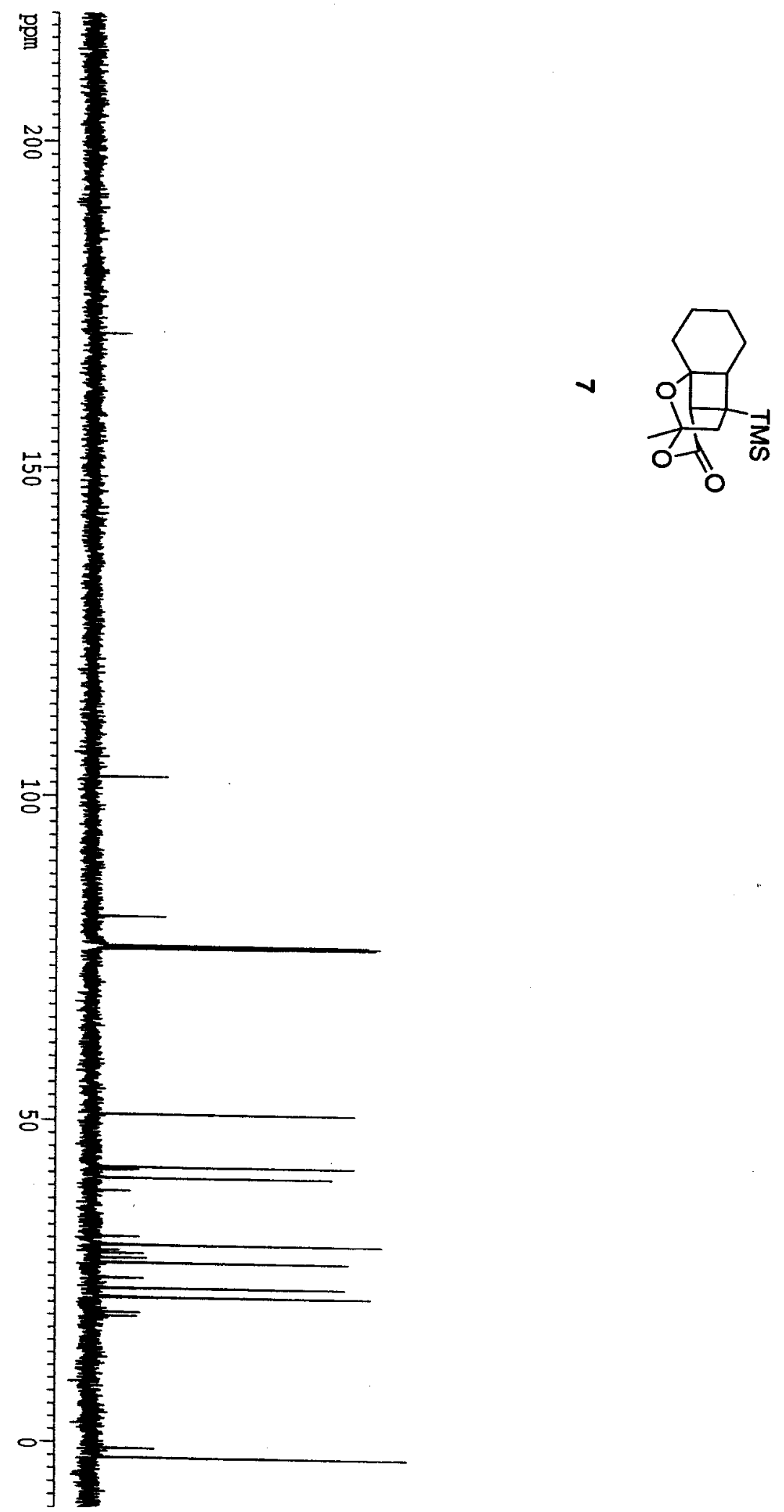

S-14 


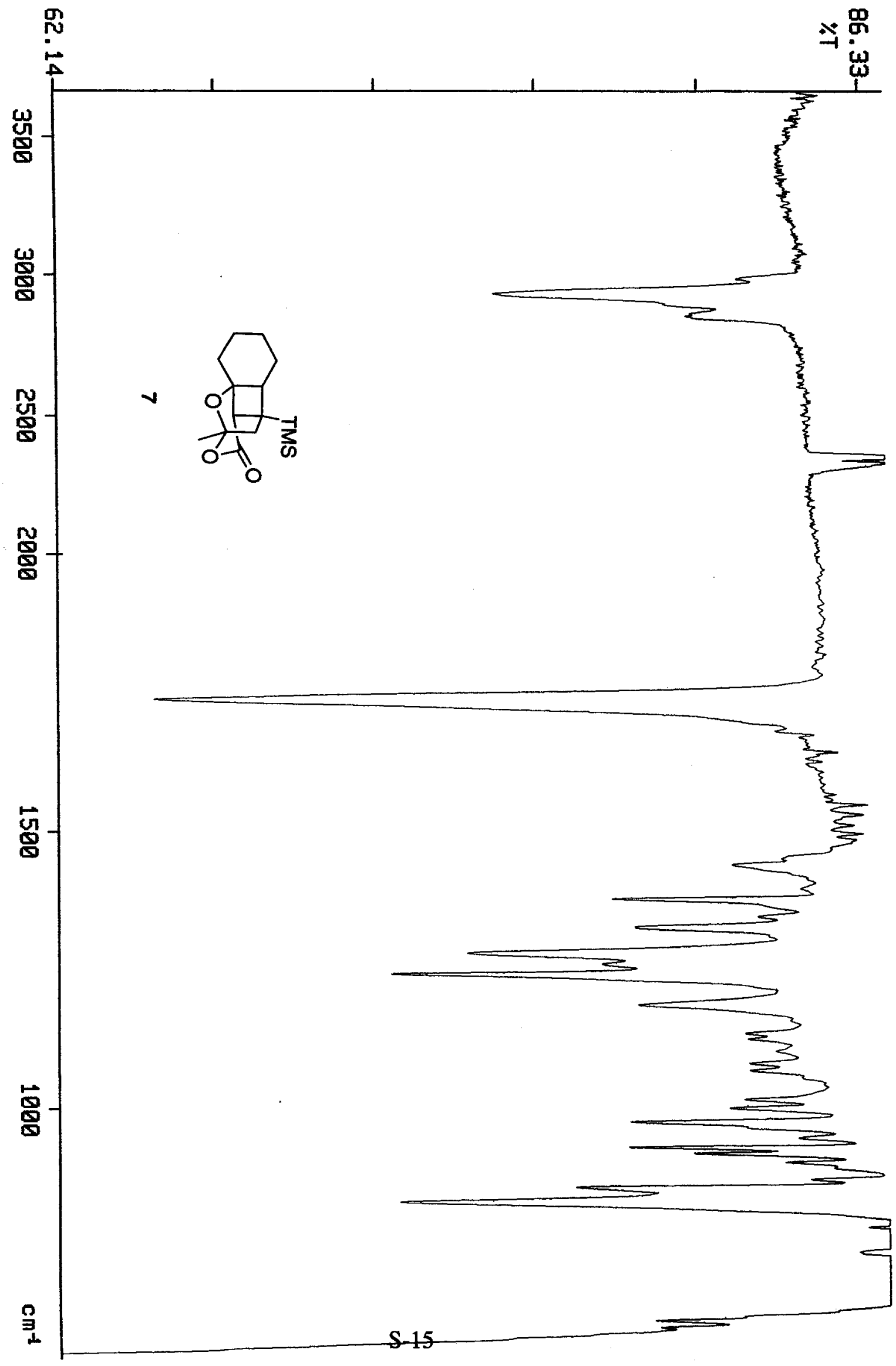




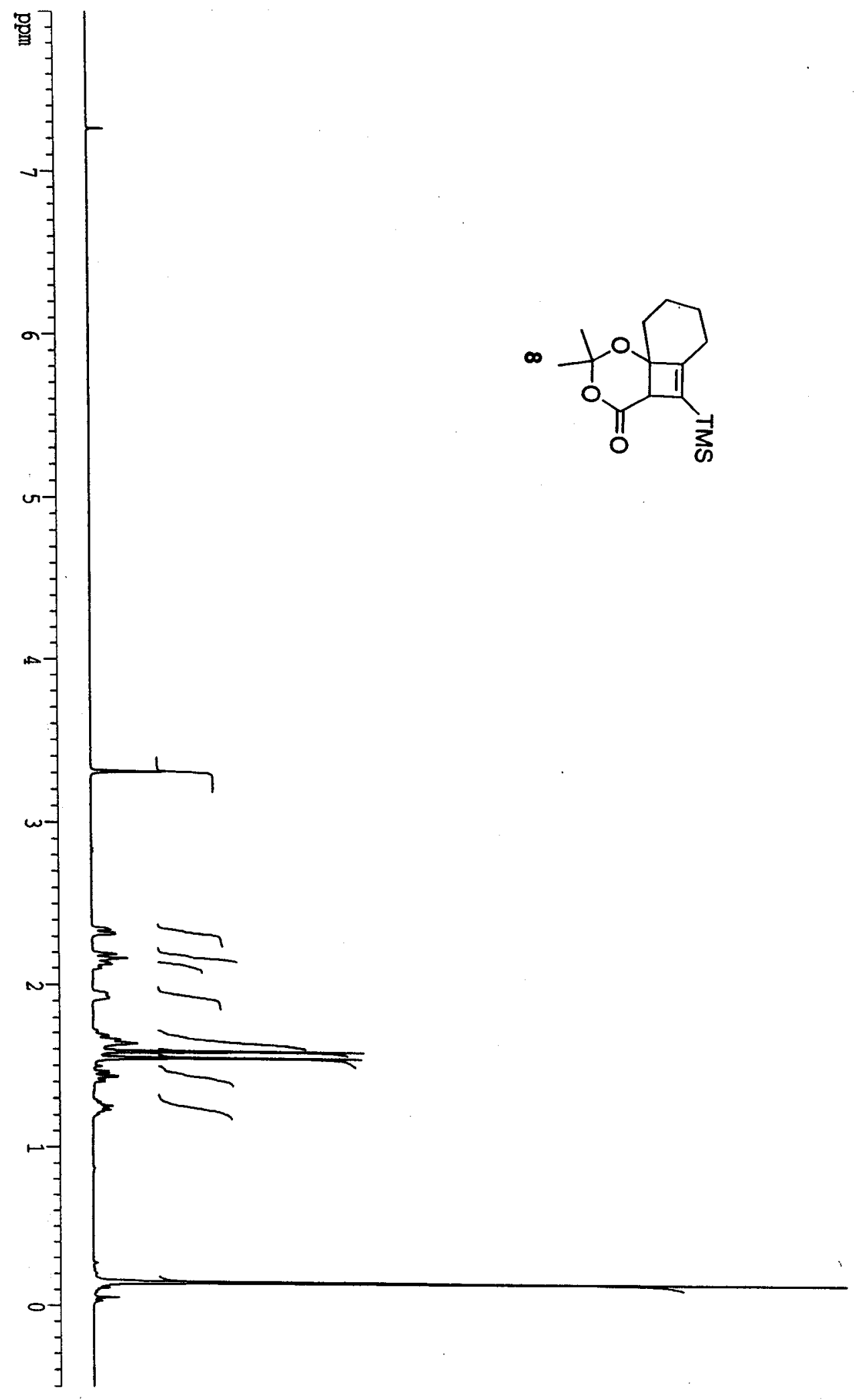



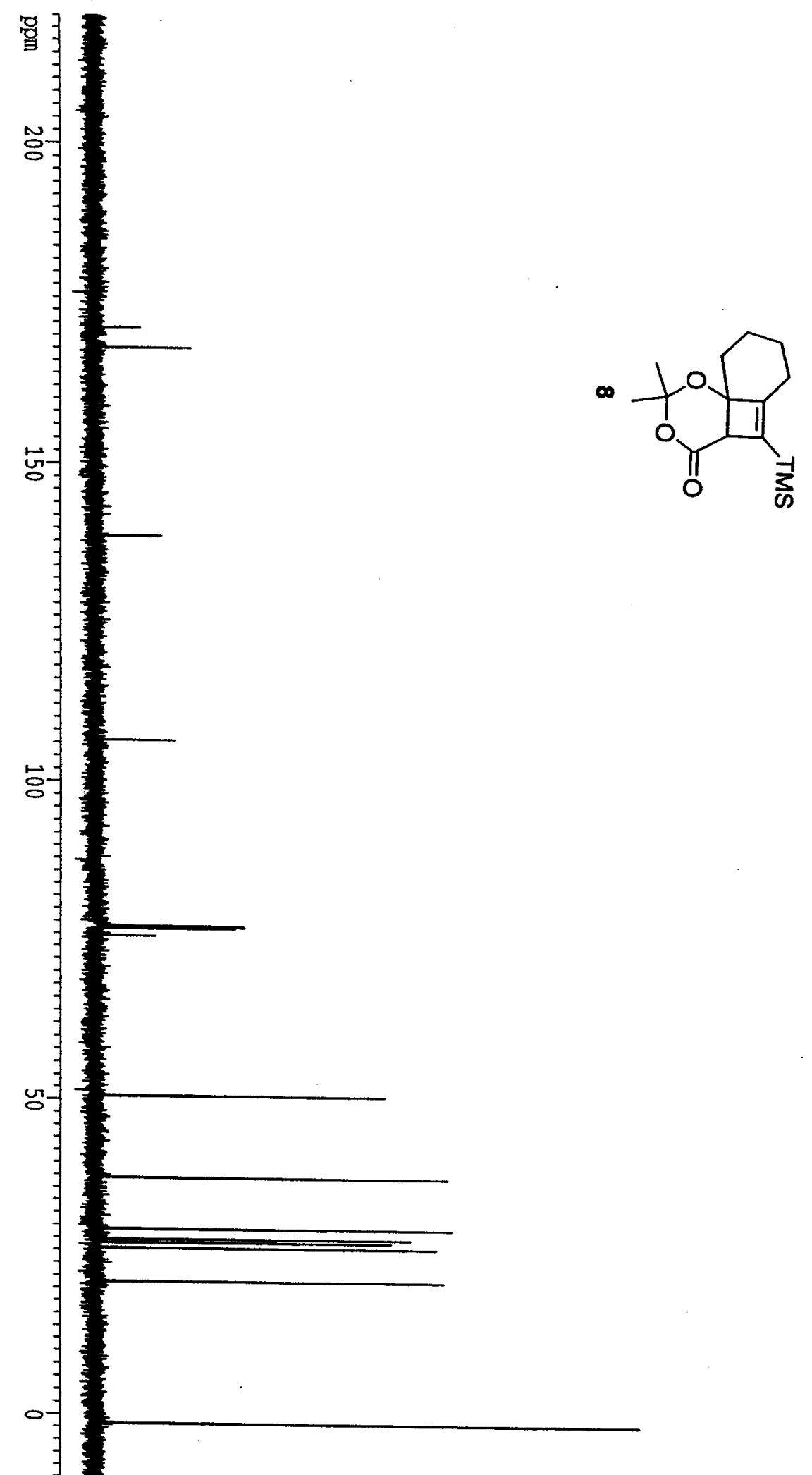

S-17 


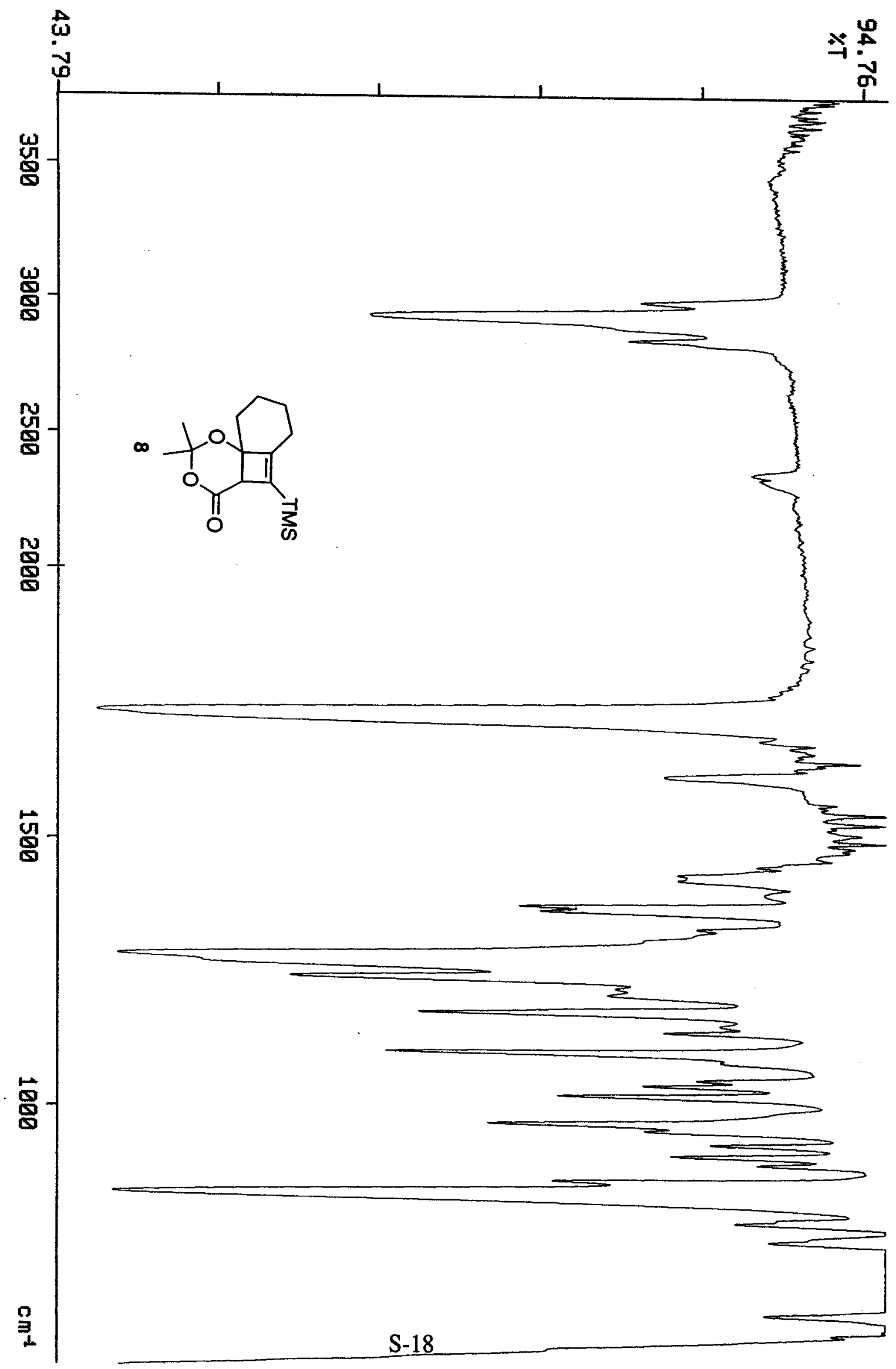




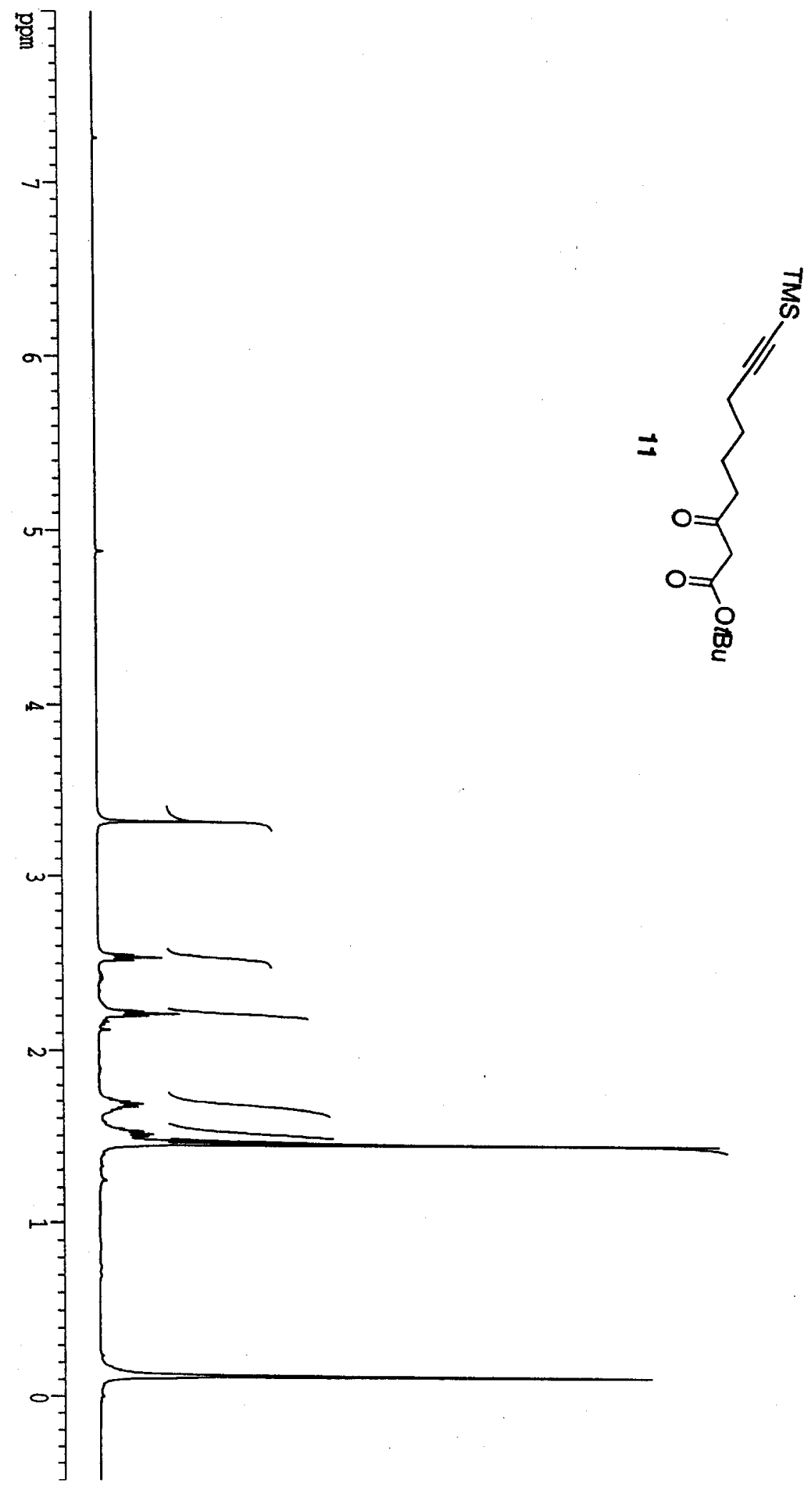




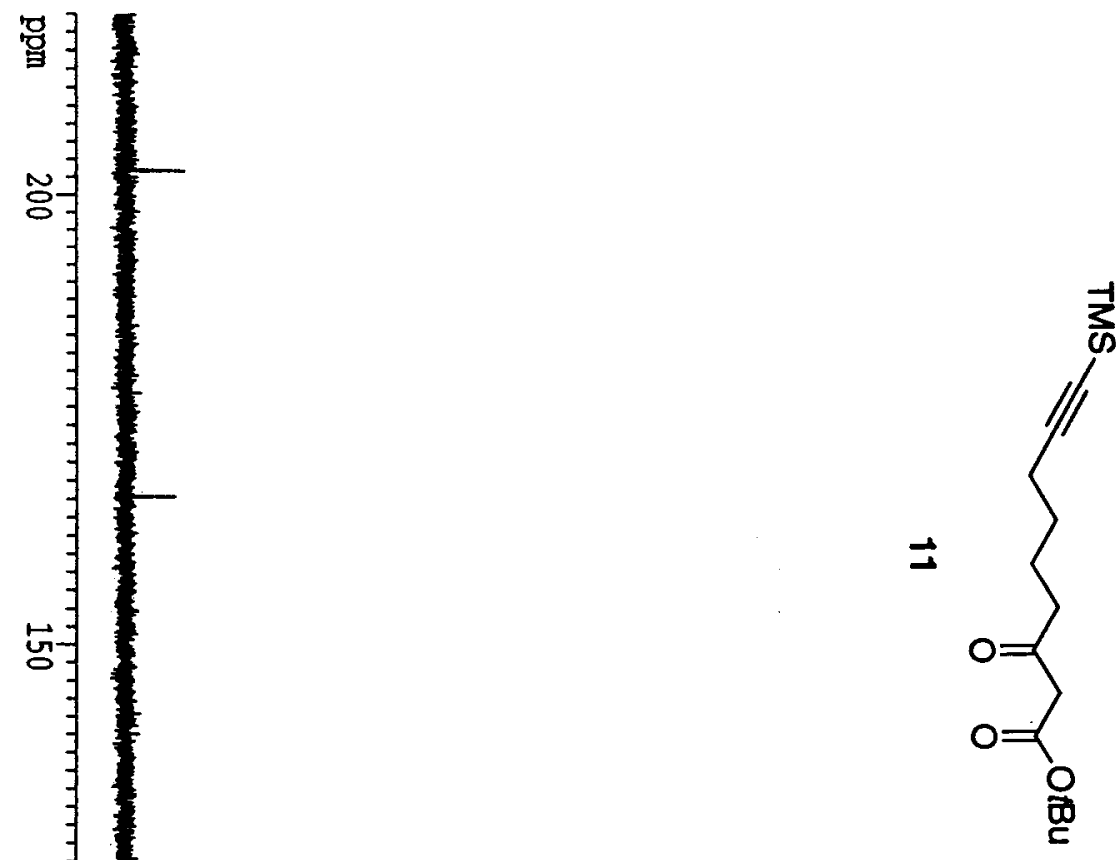




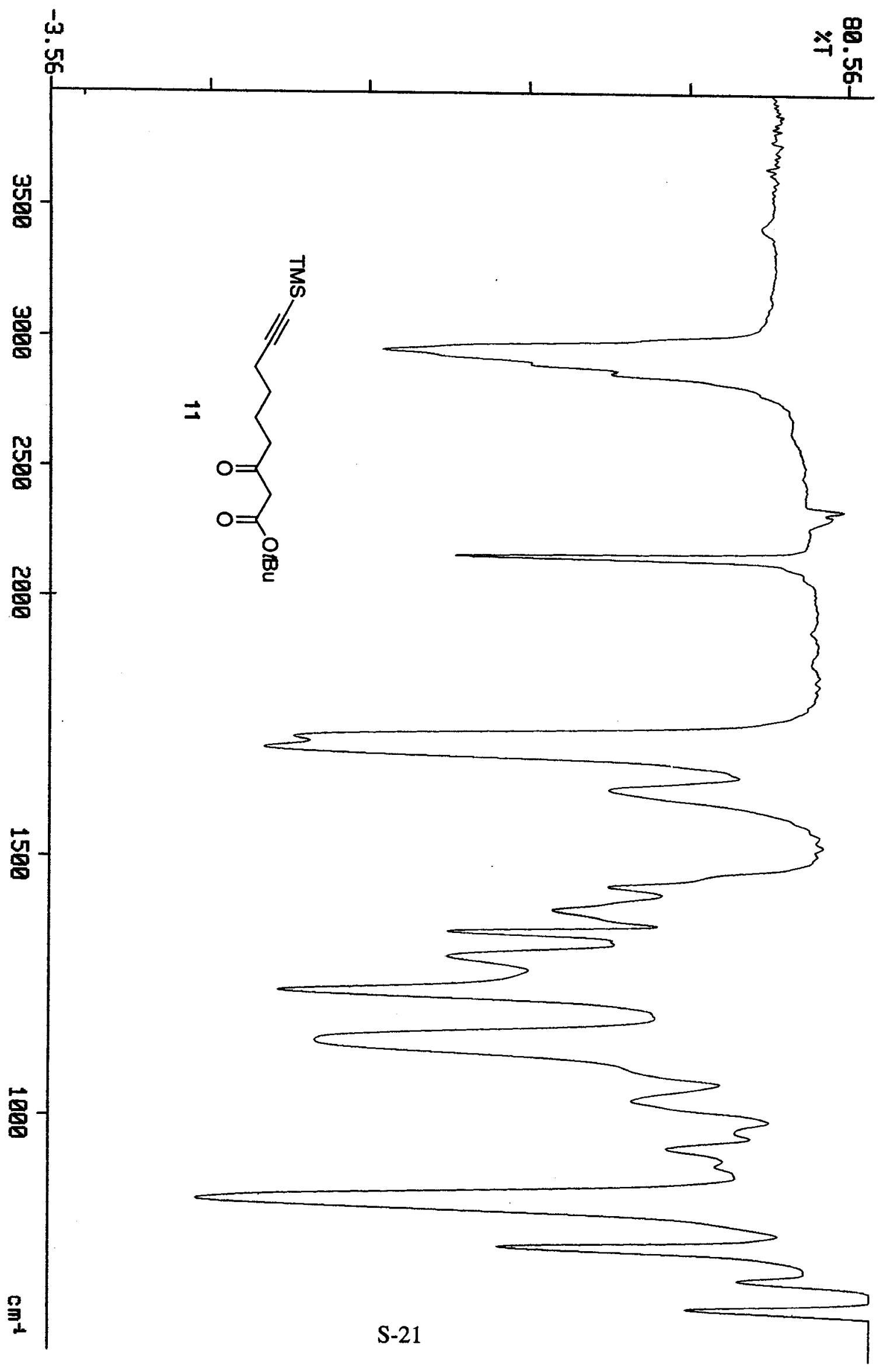




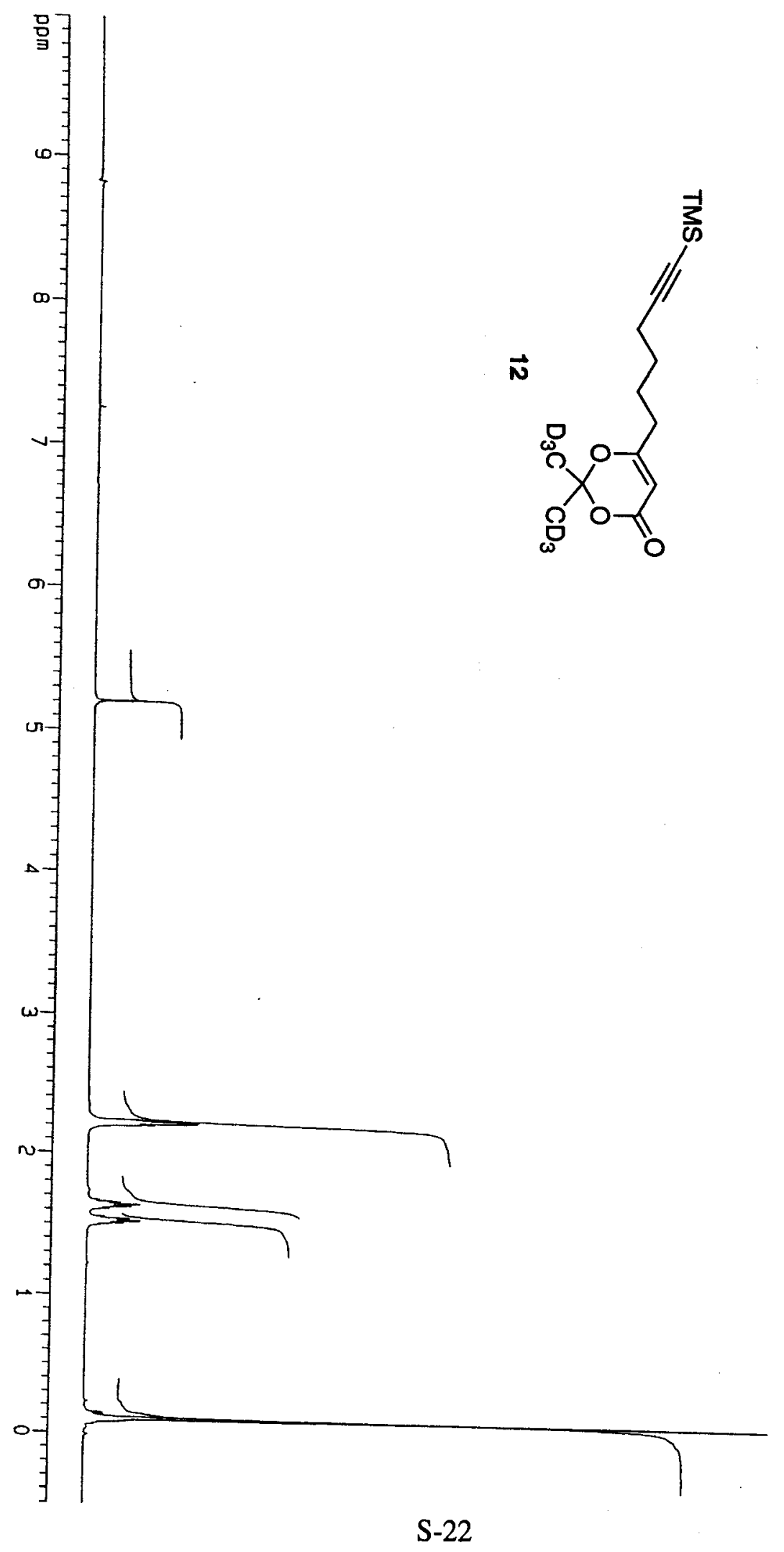




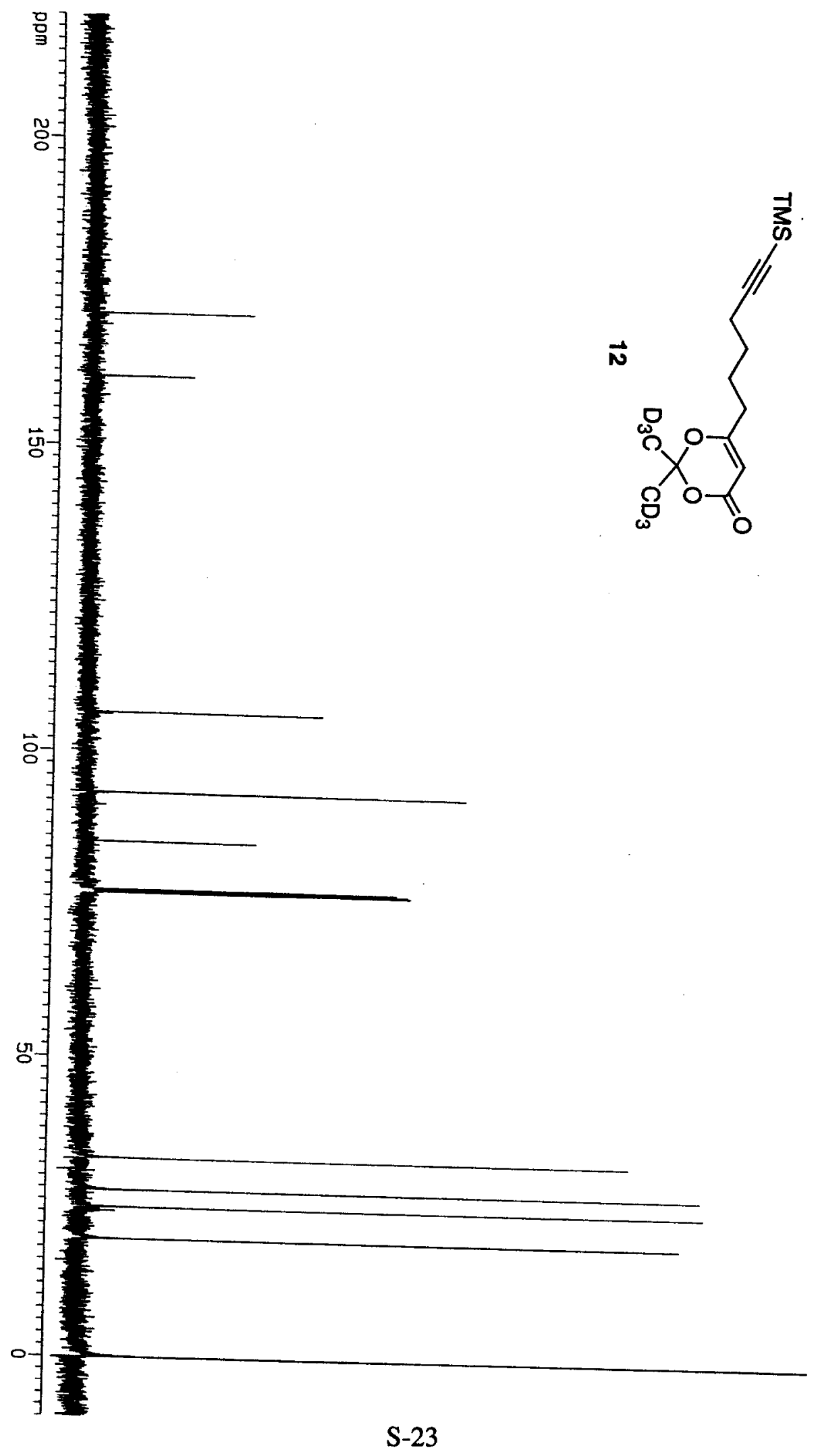




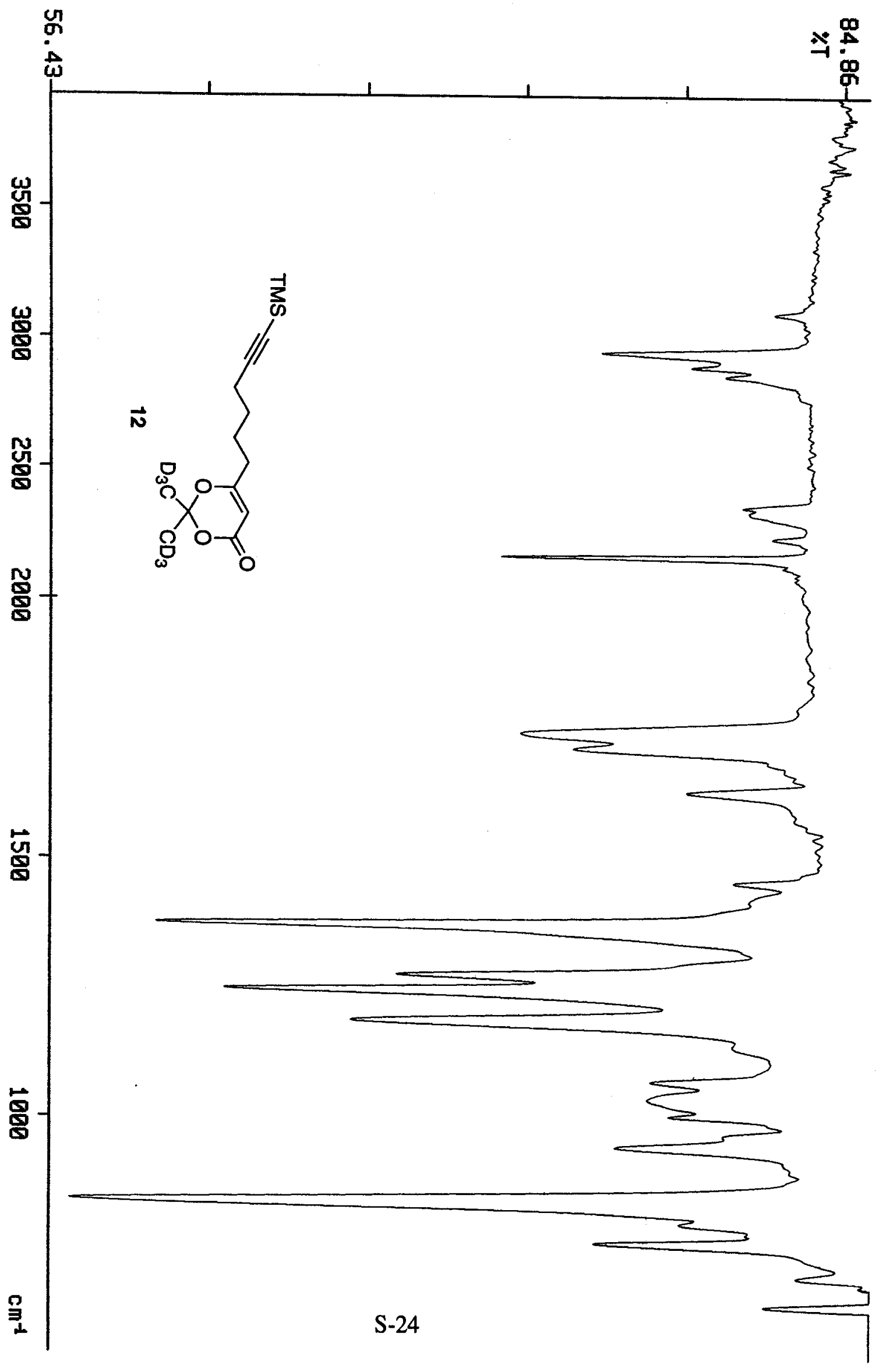




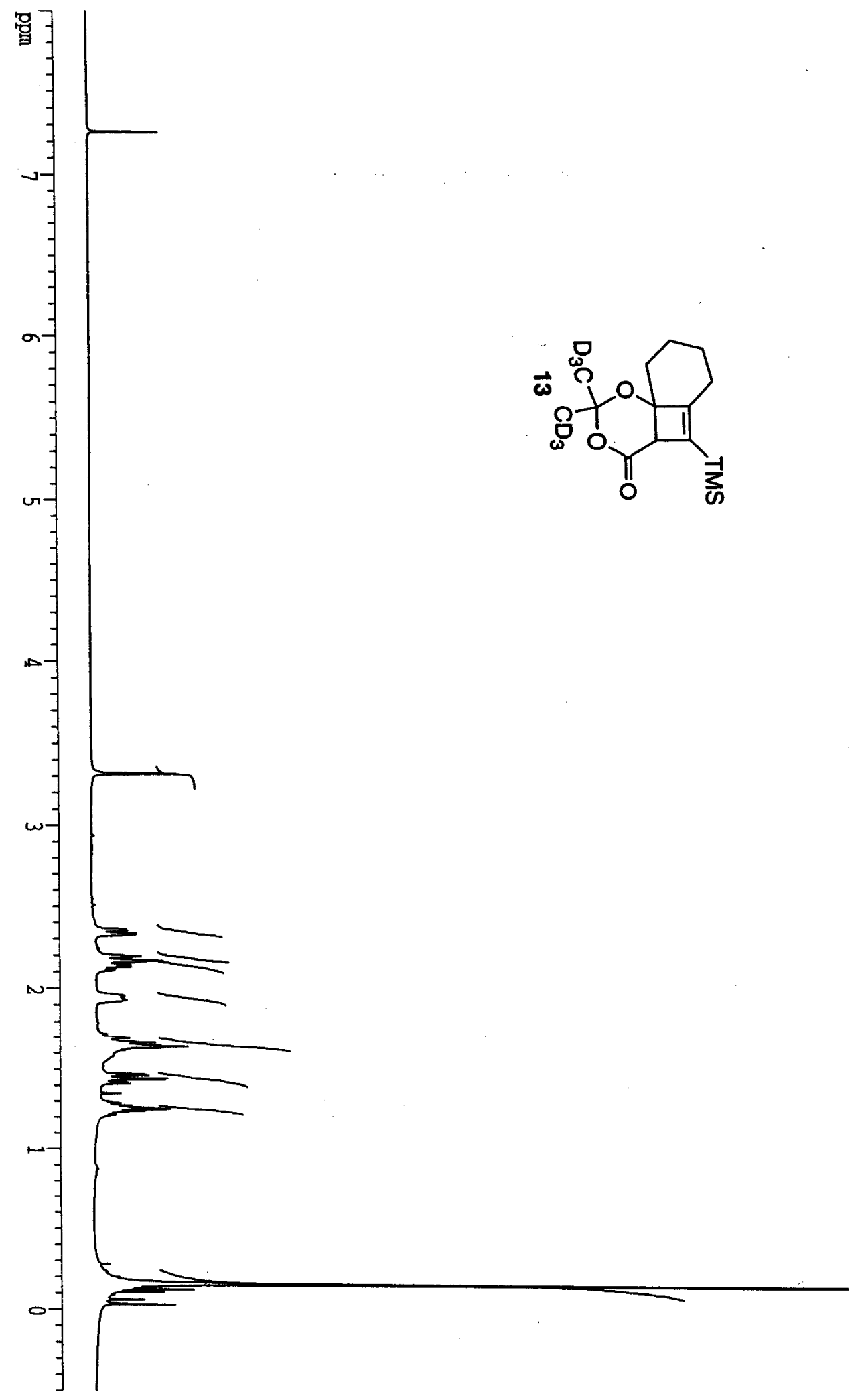




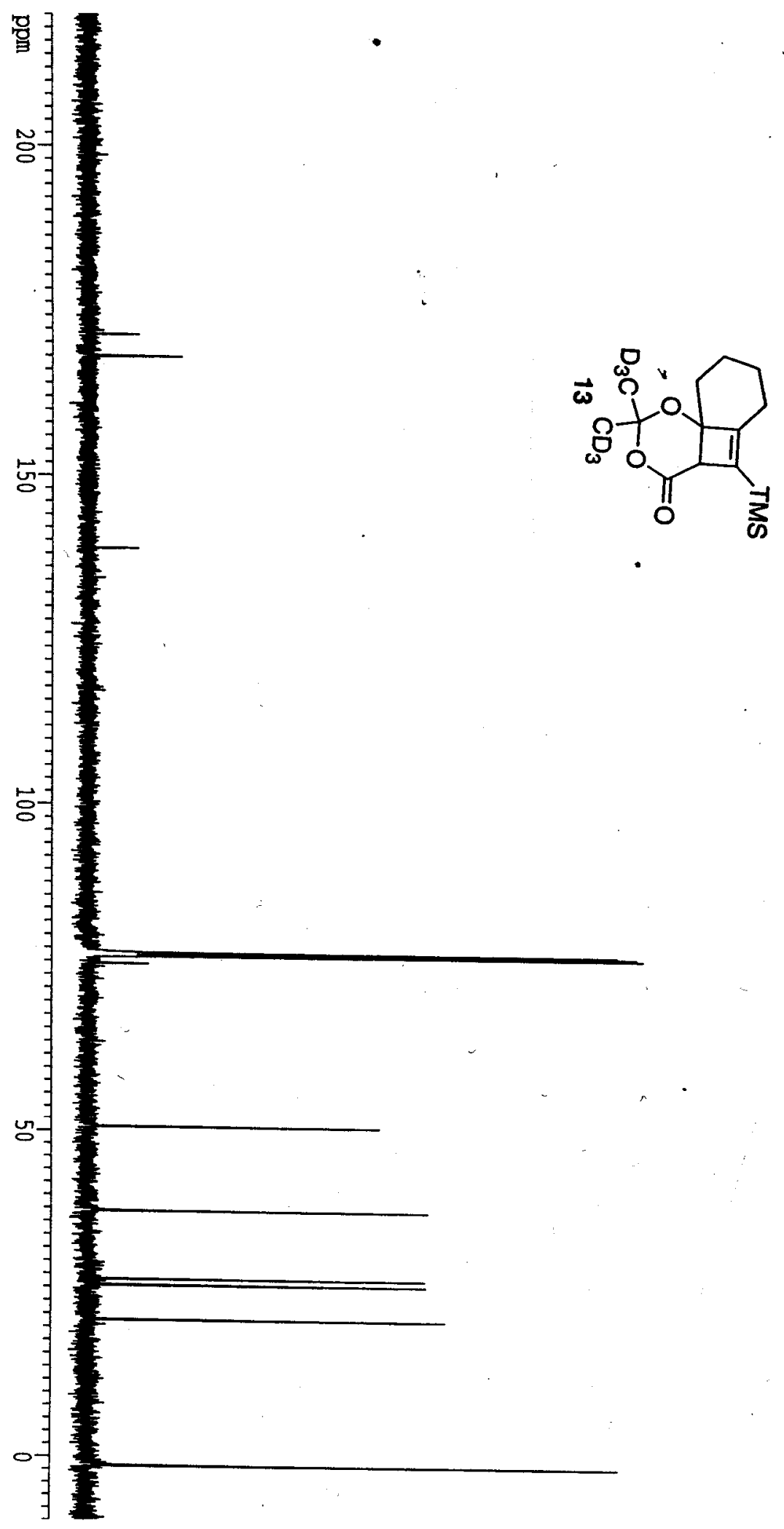

S-26 


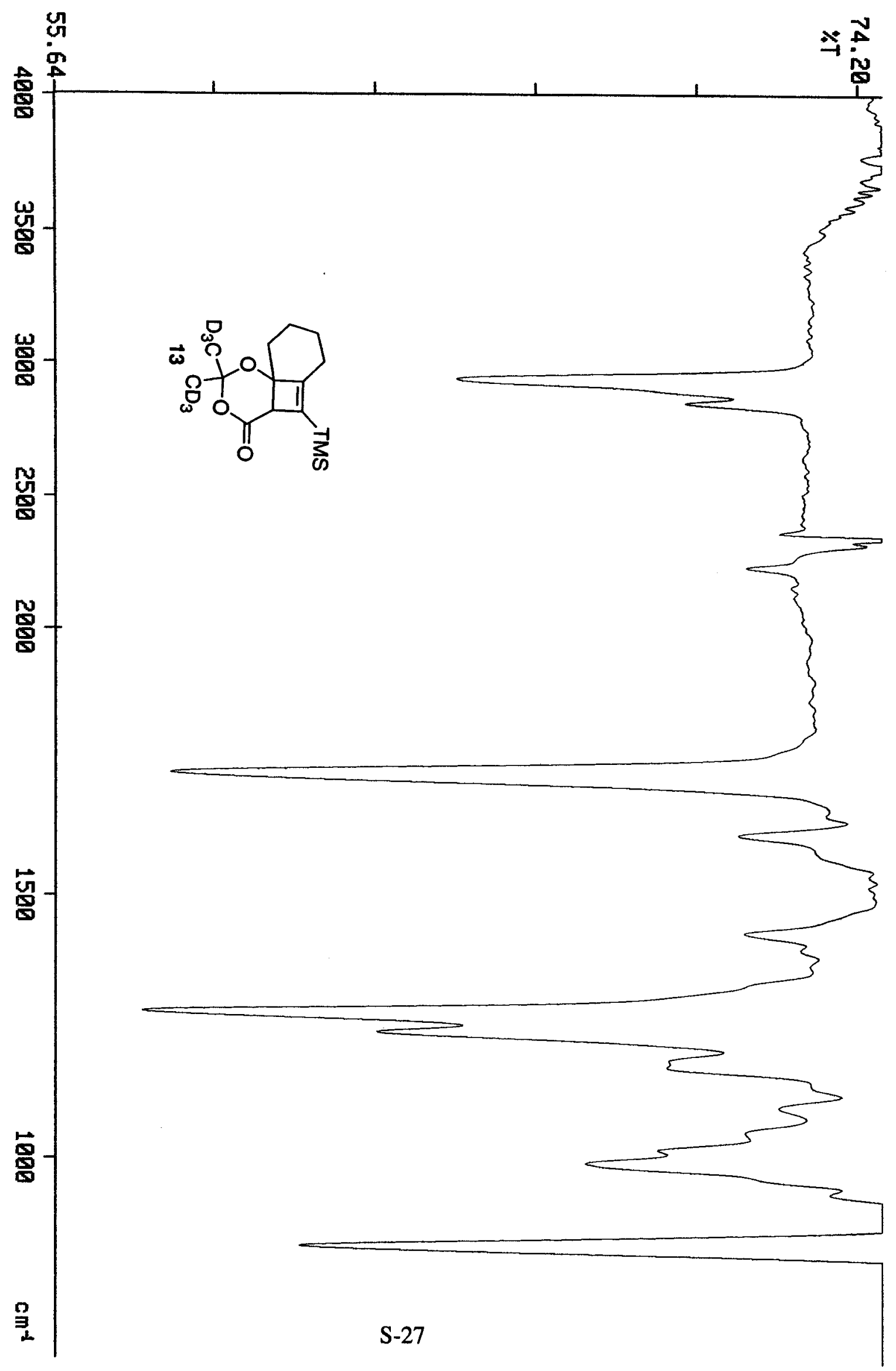




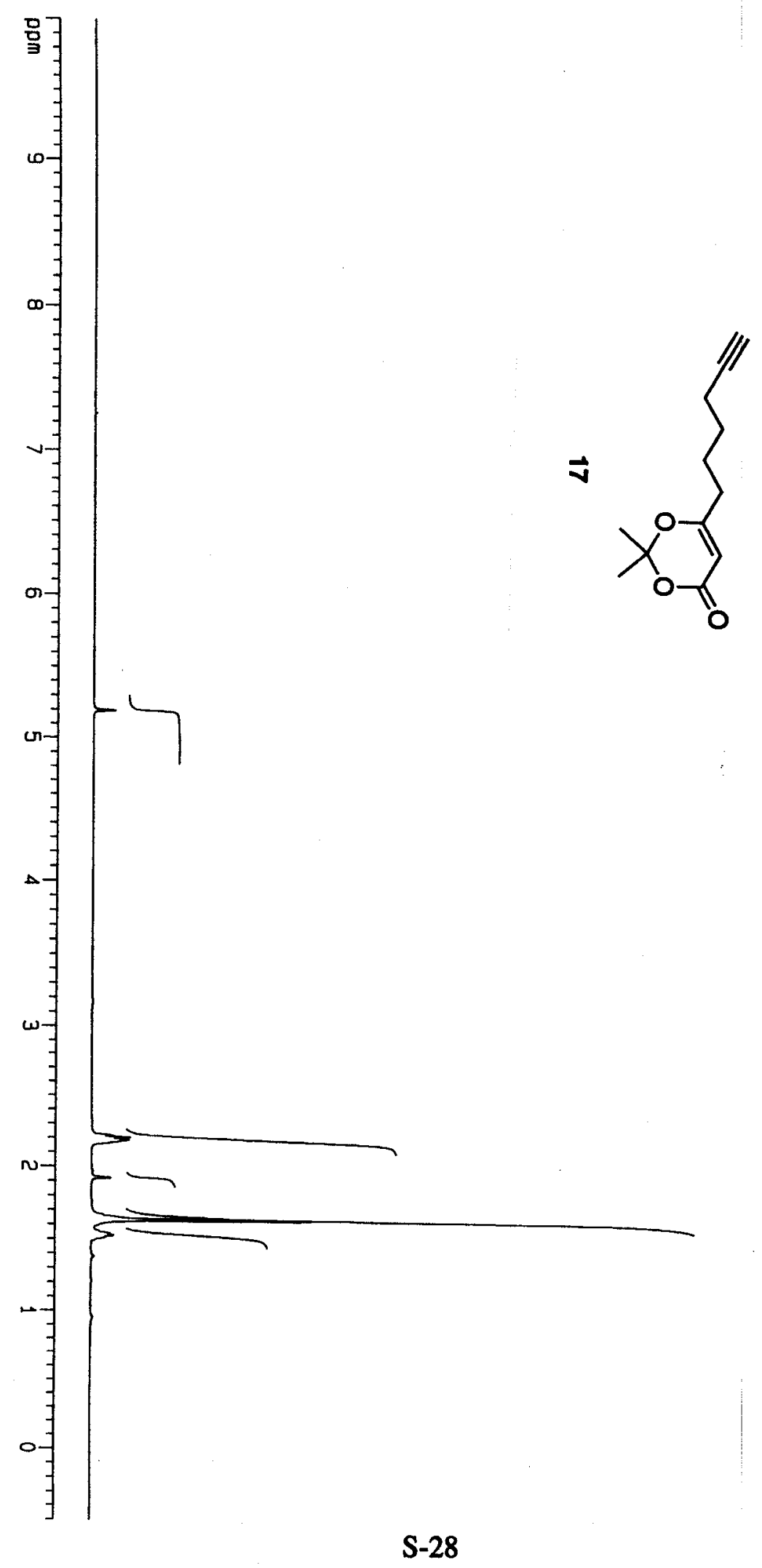




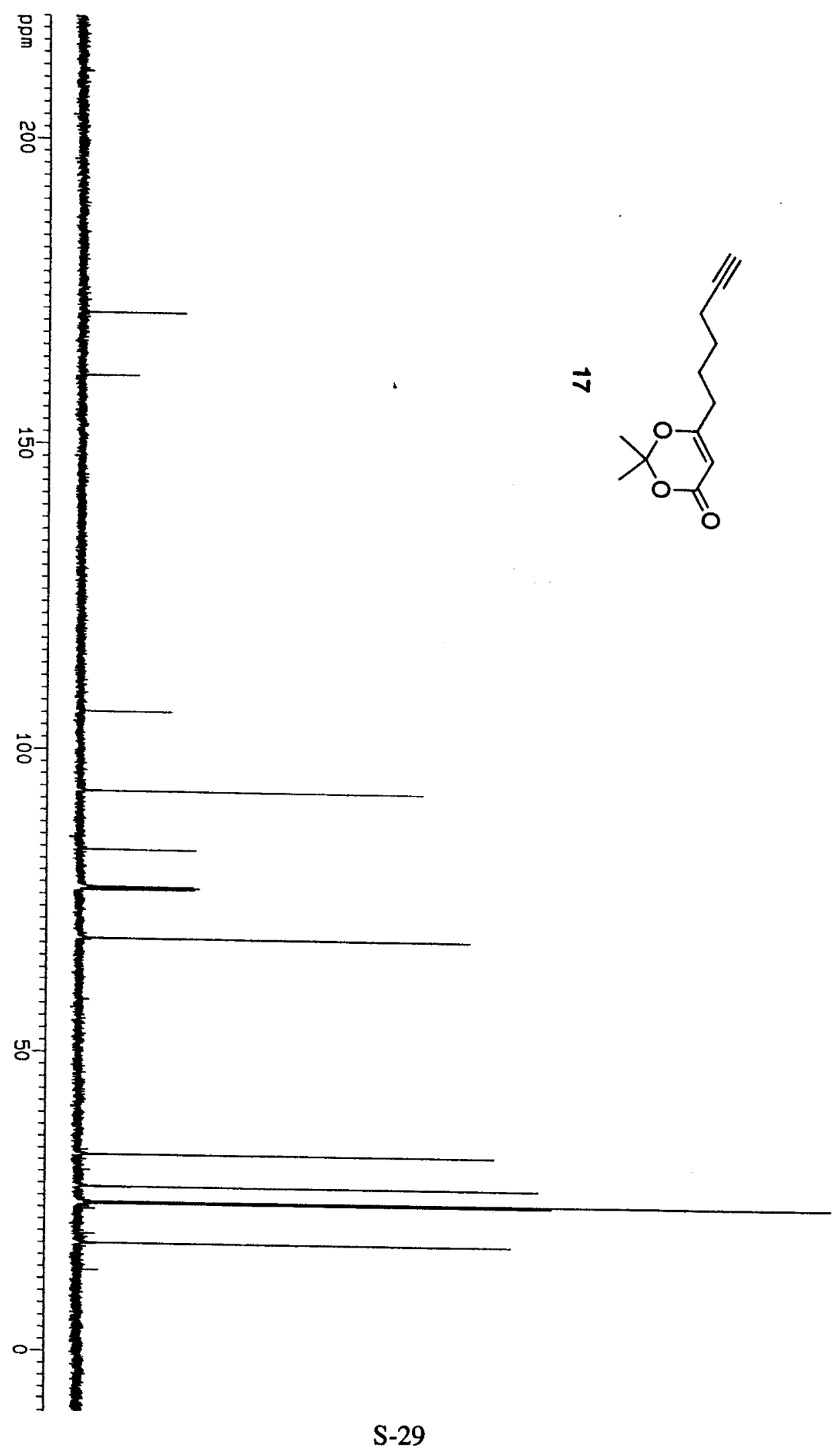




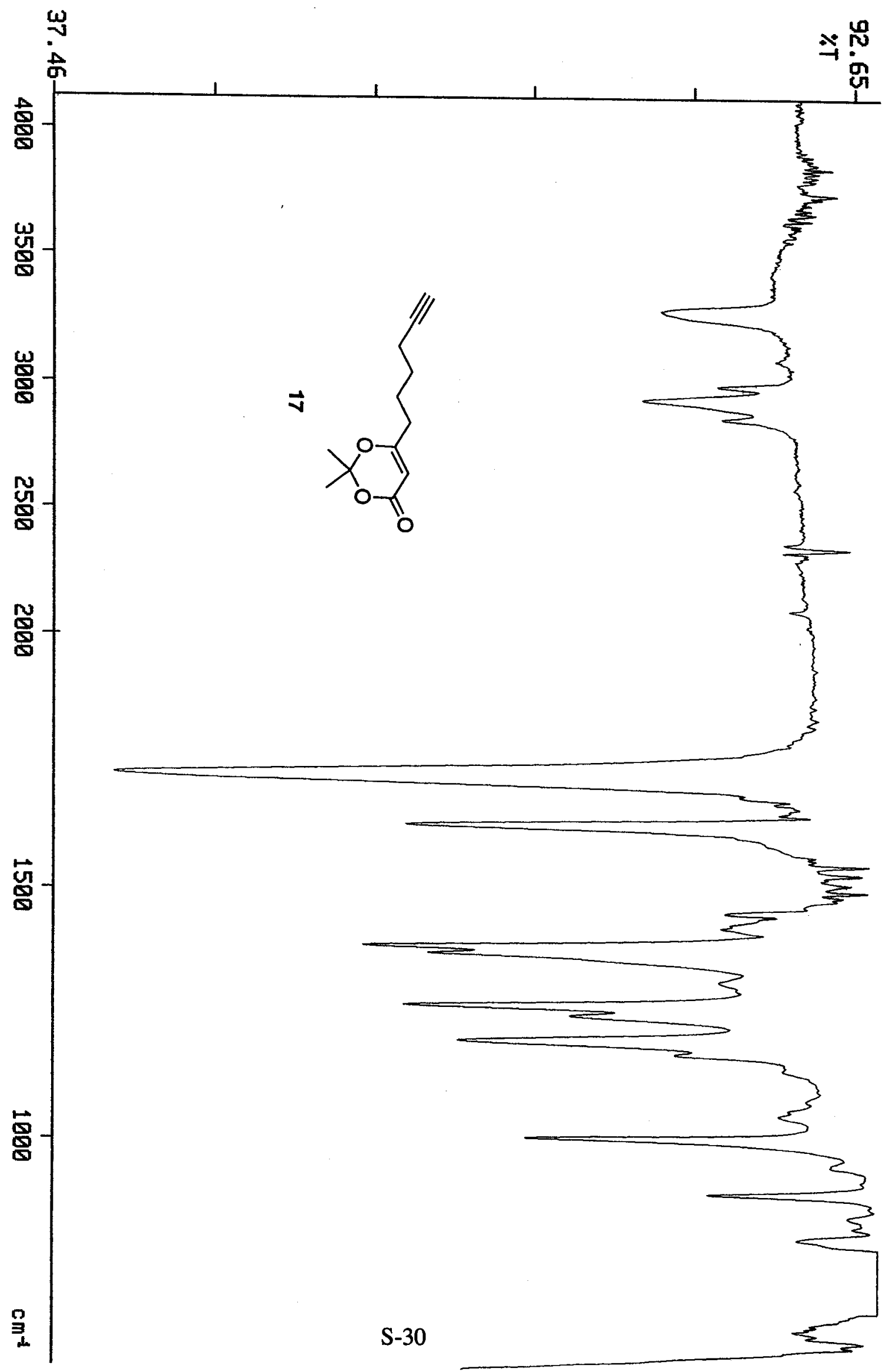




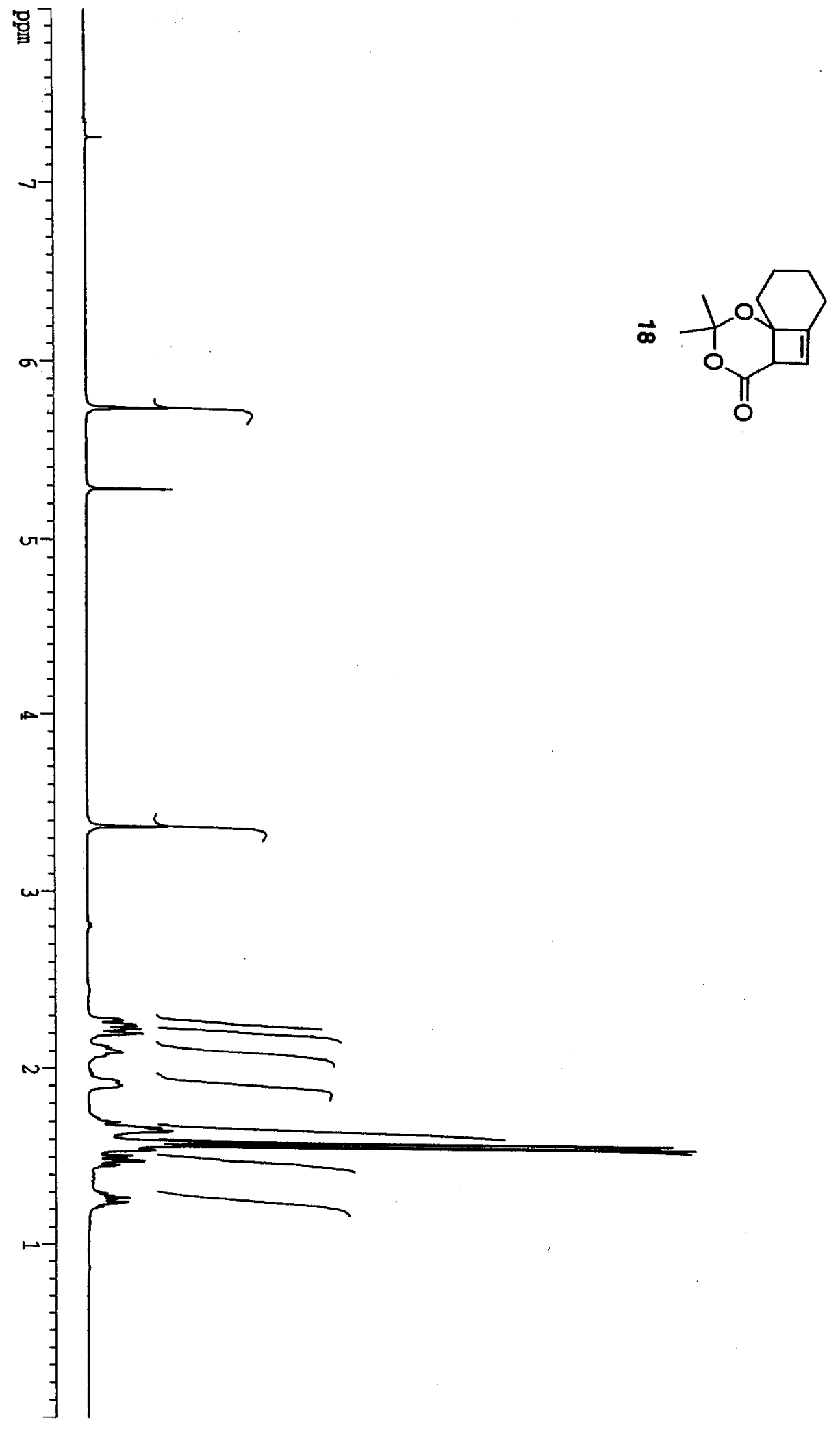



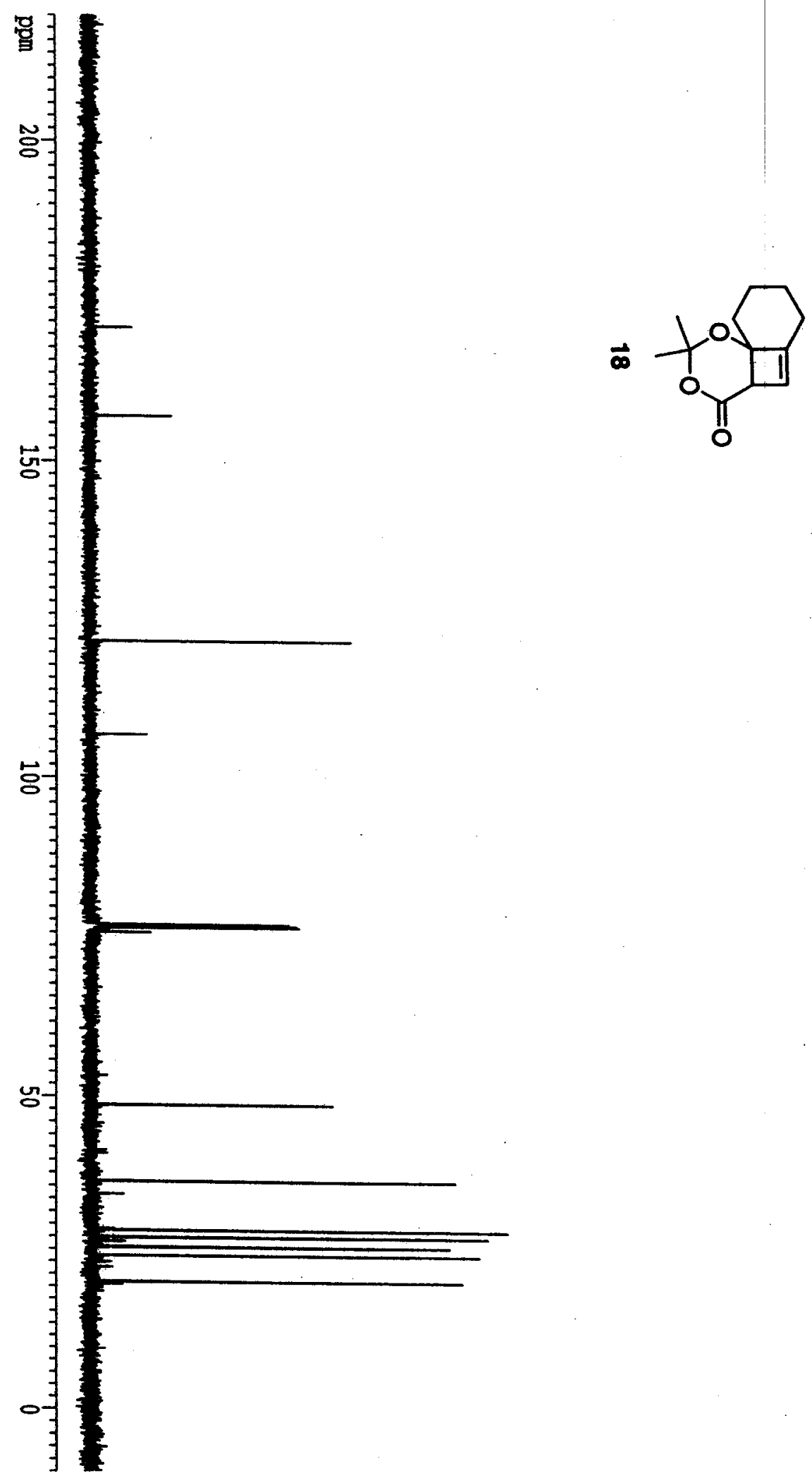

S-32 


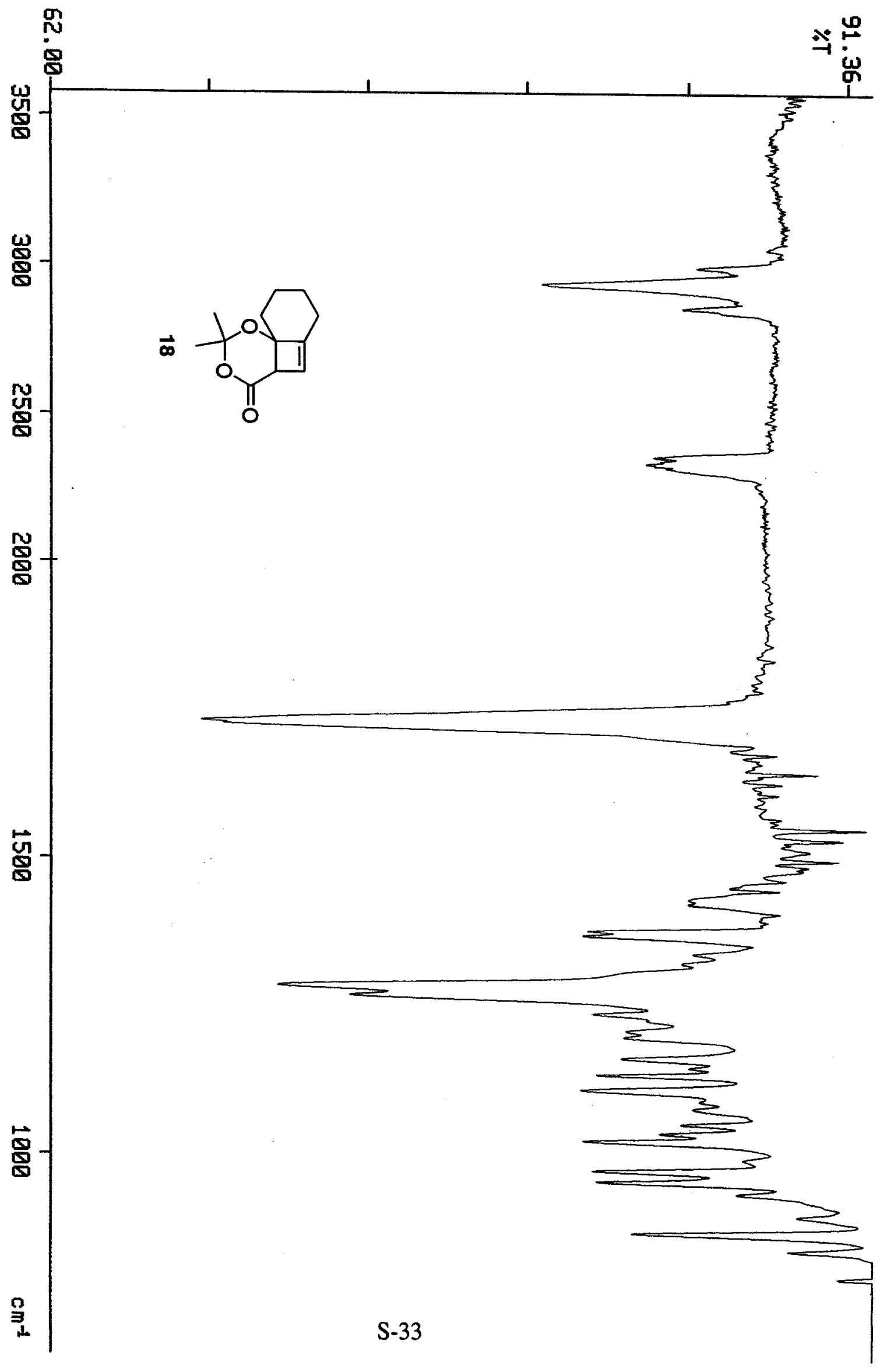




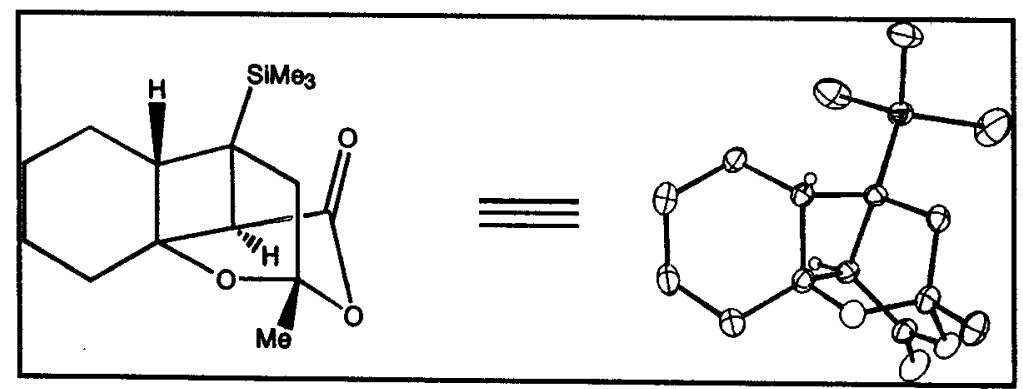

Compound 2023, $\mathrm{C}_{15} \mathrm{H}_{24} \mathrm{SiO}_{3}$, crystallizes in the monoclinic space group $\mathrm{P} 2 / \mathrm{n}$ (systematic absences Ok0: $k=$ odd and $h 0 l: h+l=0 d d)$ with $a=8.9500(8) \AA, b=15.8084(11) \AA, c=11.8213(10) \AA$, $\beta=110.1640(10)^{\circ}, V=1570.0(2) \AA^{3}, Z=4$ and $d_{c a l c}=1.186 \mathrm{~g} / \mathrm{cm}^{3}$. X-ray intensity data were collected on $a$ Rigaku Mercury CCD area detector employing graphite-monochromated Mo- $K_{\alpha}$ radiation $(\lambda=0.71069 \AA)$ at a temperature of $143^{\circ} \mathrm{K}$. Preliminary indexing was performed from a series of twelve $0.5^{\circ}$ rotation images with exposures of 30 seconds. A total of $\mathbf{4 4 0}$ rotation images were collected with a crystal to detector distance of $36 \mathrm{~mm}$, a $2 \theta$ swing angle of $-10^{\circ}$, rotation widths of $0.5^{\circ}$ and exposures of 75 seconds: scan no. 1 was a $\phi$-scan from $135^{\circ}$ to $315^{\circ}$ at $\omega=10^{\circ}$ and $\chi=20^{\circ}$ and scan no. 2 was an $\omega-$ scan from $-20^{\circ}$ to $20^{\circ}$ at $\chi=-90^{\circ}$ and $\phi=225^{\circ}$. Rotation images were processed using CrystalClear', producing a listing of unaveraged $F^{2}$ and $\sigma\left(F^{2}\right)$ values which were then passed to the Crystalstructure ${ }^{2}$ program package for further processing and structure solution on a Dell Pentium III computer. A total of 8552 reflections were measured over the ranges $5.16 \leq 20 \leq 50.66^{\circ},-10 \leq h \leq 8,-19 \leq k \leq 18,-14 \leq$ । $\leq 12$ yielding 2812 unique reflections $\left(R_{\mathrm{nt}}=0.0230\right)$. The intensity data were corrected for Lorentz and polarization effects and for absorption. using REQAB ${ }^{3}$ (minimum and maximum transmission 0.803, 1.000).

The structure was solved by direct methods (SIR97 ${ }^{4}$ ). Refinement was by full-matrix least squares based on $F^{2}$ using SHELXL-97 $7^{5}$. All reflections were used during refinement ( $F^{2}$ 's that were experimentally negative were replaced by $F^{2}=0$ ). The weighting scheme used was $w=1 /\left[\sigma^{2}\left(F_{0}^{2}\right)+0.1618 P^{2}+0.5002 P\right]$ where $P=\left(F_{0}^{2}+2 F_{c}^{2}\right) / 3$. Non-hydrogen atoms were refined anisotropically and hydrogen atoms were refined using a "riding" model. Refinement converged to $R_{1}=0.0557$ and $w R_{2}=0.1803$ for 2391 reflections 
for which $F>4 \sigma(F)$ and $R_{1}=0.0742, w R_{2}=0.2284$ and $G O F=1.081$ for all 2812 unique, non-zero reflections and 177 variables $^{6}$. The maximum $\Delta / \sigma$ in the final cycle of least squares was 0.000 and the two most prominent peaks in the final difference Fourier were +0.538 and $-0.520 \mathrm{e} / \AA^{3}$.

Table 1. lists cell information, data collection parameters, and refinement data. Final positional and equivalent isotropic thermal parameters are given in Table 2. Anisotropic thermal parameters are in Table 3. Tables 4. and 5. list bond distances and bond angles. Figure 1. is an ORTEP ${ }^{7}$ representation of the molecule with $30 \%$ probability thermal ellipsoids displayed.

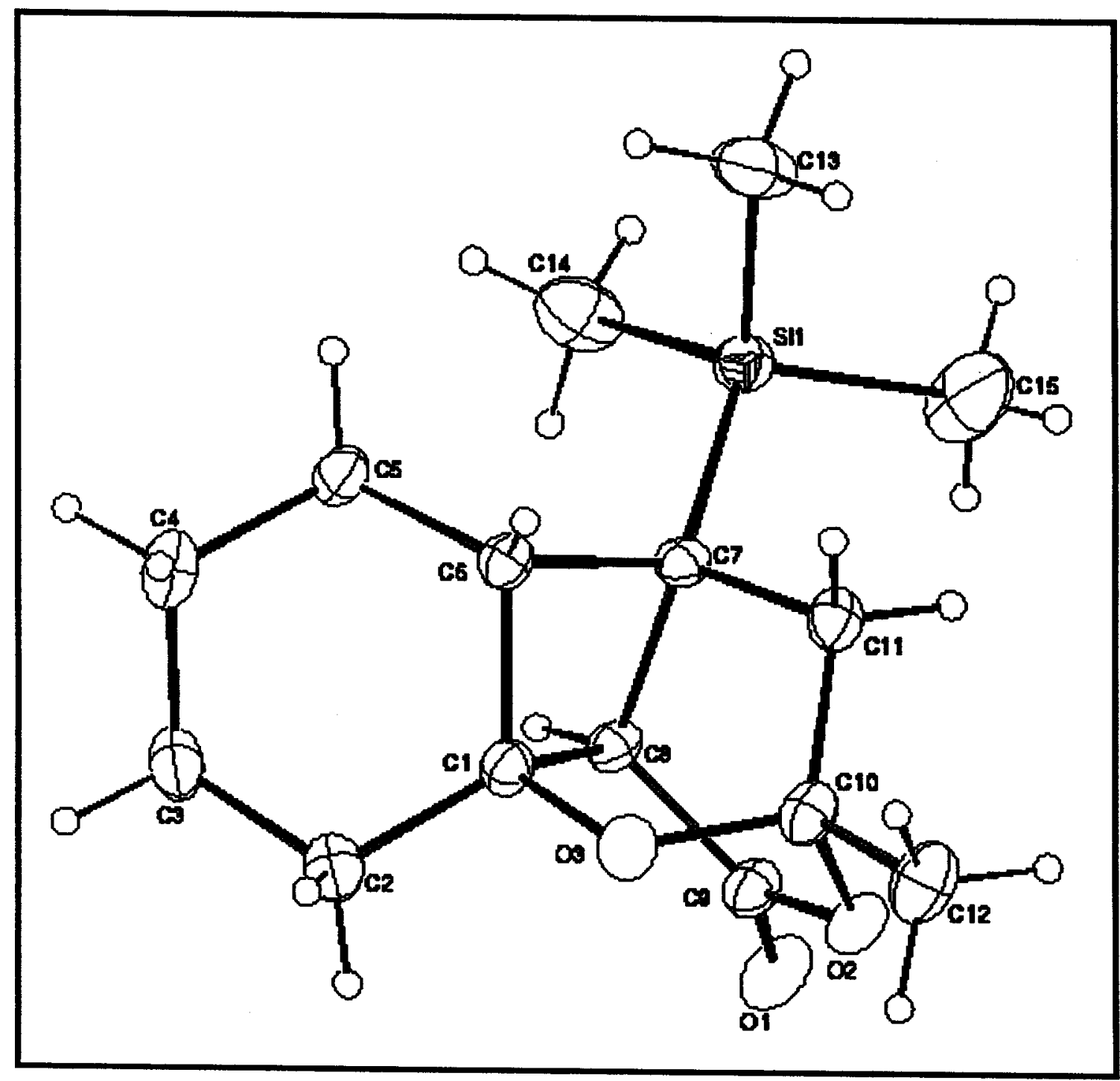

Figure 1. ORTEP drawing of the title compound with $30 \%$ probability thermal ellipsoids. 


\section{References}

1. CrystalClear: Rigaku Corporation, 1999.

2. EnystalStructure: Crystal Structure Analysis Package, Rigaku Corp. Rigaku/MSC (2002).

3. REQAB4: R.A. Jacobsen, (1994). Private Communication.

4. SIR97: Altomare, A., M. Burla, M. Camalli, G. Cascarano, C. Giacovazzo, A. Guagliardi, A. Moliterni, G. Polidori \& R. Spagna (1999). J. Appl. Cryst, 32, 115-119.

5. SHELXL-97: Program for the Refinement of Crystal Structures, Sheldrick, G.M. (1997), University of Göttingen, Germany.

6. $R_{1}=\sum\left\|F_{0}|-| F_{C}\right\| / \sum\left|F_{0}\right|$

$w R_{2}=\left\{\sum w\left(F_{0}^{2}-F_{c}^{2}\right)^{2} / \sum w\left(F_{0}^{2}\right)^{2}\right\}^{1 / 2}$

GOF $=\left\{\sum w\left(F_{0}^{2}-F_{c}^{2}\right)^{2} /(n-p)\right\}^{1 / 2}$

where $n=$ the number of reflections and $p=$ the number of parameters refined.

7. "ORTEP-II: A Fortran Thermal Ellipsoid Plot Program for Crystal Structure Illustrations". C.K. Johnson (1976) ORNL-5138. 


\section{Table 1. Summary of Structure Determination of Compound 2023}

Formula:

Formula weight:

Crystal class:

Space group:

Z

Cell constants:
a
b
c
$\beta$
V

$\mu$

crystal size, $\mathrm{mm}$

$D_{\text {calc }}$

$F(000)$

Radiation:

$2 \theta$ range

hkl collected:

No. reflections measured:

No. unique reflections:

No. observed reflections

No. reflections used in refinement

No. parameters

$R$ indices ( $F>4 \sigma$ )

$R$ indices (all data)

GOF:

Final Difference Peaks, el $\AA^{3}$
$\mathrm{C}_{15} \mathrm{H}_{24} \mathrm{SiO}_{3}$

280.43

monoclinic

P2,/n (\#14)

4

$8.9500(8) \AA$

$15.8084(11) \AA$

$11.8213(10) \AA$

$110.1640(10)^{\circ}$

$1570.0(2) \dot{A}^{3}$

$1.52 \mathrm{~cm}^{-1}$

$0.35 \times 0.32 \times 0.04$

$1.186 \mathrm{~g} / \mathrm{cm}^{3}$

608

Mo- $K_{\alpha}(\lambda=0.71069 \AA)$

$5.16-50.66^{\circ}$

$-10 \leq h \leq 8 ;-19 \leq k \leq 18 ;-14 \leq \mid \leq 12$

8552

$2812\left(\mathrm{R}_{\mathrm{im}}=0.0230\right)$

2391 ( $F>4 \sigma)$

2812

177

$R_{1}=0.0557$

$w_{2}=0.1803$

$R_{1}=0.0742$

$w R_{2}=0.2284$

1.081

$+0.538,-0.520$ 
Table 2. Refined Positional Parameters for Compound 2023

\begin{tabular}{|c|c|c|c|c|}
\hline Atom & $x$ & $y$ & $\mathbf{z}$ & $U_{\theta q}, \AA^{2}$ \\
\hline Si1 & $0.09633(8)$ & $0.85338(5)$ & $0.33930(7)$ & $0.0368(3)$ \\
\hline $\mathrm{C} 1$ & $0.4767(3)$ & $0.8923(2)$ & $0.2526(2)$ & $0.0305(6)$ \\
\hline $\mathrm{C} 2$ & $0.5680(4)$ & $0.9587(2)$ & $0.2118(3)$ & $0.0456(7)$ \\
\hline $\mathrm{H} 2 \mathrm{a}$ & 0.6012 & 0.9354 & 0.1483 & 0.061 \\
\hline $\mathrm{H} 2 \mathrm{~b}$ & 0.6630 & 0.9736 & 0.2787 & 0.061 \\
\hline $\mathrm{C} 3$ & $0.4695(4)$ & $1.0385(2)$ & $0.1654(3)$ & $0.0463(8)$ \\
\hline $\mathrm{H} 3 \mathrm{a}$ & 0.4588 & 1.0696 & 0.2329 & 0.062 \\
\hline $\mathrm{H} 3 \mathrm{~b}$ & 0.5245 & 1.0745 & 0.1260 & 0.062 \\
\hline $\mathrm{C} 4$ & $0.3050(4)$ & $1.0173(2)$ & $0.0769(3)$ & $0.0450(7)$ \\
\hline $\mathrm{H} 4 \mathrm{a}$ & 0.2469 & 1.0690 & 0.0459 & 0.060 \\
\hline $\mathrm{H} 4 \mathrm{~b}$ & 0.3147 & 0.9856 & 0.0095 & 0.060 \\
\hline C5 & $0.2152(3)$ & $0.9650(2)$ & $0.1405(2)$ & $0.0358(6)$ \\
\hline $\mathrm{H} 5 \mathrm{a}$ & 0.1079 & 0.9546 & 0.0856 & 0.048 \\
\hline H5b & 0.2079 & 0.9965 & 0.2088 & 0.048 \\
\hline C6 & $0.2979(3)$ & $0.8808(2)$ & $0.1841(2)$ & $0.0292(6)$ \\
\hline $\mathrm{H} 6$ & 0.2751 & 0.8382 & 0.1200 & 0.039 \\
\hline C7 & $0.2864(3)$ & $0.8444(2)$ & $0.3051(2)$ & $0.0272(6)$ \\
\hline C8 & $0.4399(3)$ & $0.8994(2)$ & $0.3724(2)$ & $0.0277(6)$ \\
\hline $\mathrm{H} 8$ & 0.4134 & 0.9572 & 0.3884 & 0.037 \\
\hline $\mathrm{C} 9$ & $0.5616(3)$ & $0.8598(2)$ & $0.4793(2)$ & $0.0330(6)$ \\
\hline $\mathrm{C} 10$ & $0.5237(3)$ & $0.7537(2)$ & $0.3335(2)$ & $0.0346(6)$ \\
\hline C11 & $0.3471(3)$ & $0.7521(2)$ & $0.3139(2)$ & $0.0324(6)$ \\
\hline H11a & 0.3289 & 0.7234 & 0.3806 & 0.043 \\
\hline$H 11 b$ & 0.2903 & 0.7217 & 0.2403 & 0.043 \\
\hline $\mathrm{C} 12$ & $0.5985(4)$ & $0.6703(2)$ & $0.3213(3)$ & $0.0491(8)$ \\
\hline $\mathrm{H} 12 \mathrm{a}$ & $0.5499^{\circ}$ & 0.6493 & 0.2407 & 0.074 \\
\hline $\mathrm{H} 12 \mathrm{~b}$ & 0.5826 & 0.6303 & 0.3773 & 0.074 \\
\hline $\mathrm{H} 12 \mathrm{c}$ & 0.7104 & 0.6781 & 0.3382 & 0.074 \\
\hline $\mathrm{C}_{13}$ & $-0.0592(4)$ & $0.7998(2)$ & $0.2121(4)$ & $0.0581(9)$ \\
\hline $\mathrm{H13a}$ & -0.0257 & 0.7431 & 0.2040 & 0.087 \\
\hline $\mathrm{H} 13 \mathrm{~b}$ & -0.0745 & 0.8304 & 0.1389 & 0.087 \\
\hline $\mathrm{H} 13 \mathrm{c}$ & -0.1573 & 0.7982 & 0.2280 & 0.087 \\
\hline$C_{14}$ & $0.0369(4)$ & $0.9645(2)$ & $0.3521(4)$ & $0.0588(9)$ \\
\hline $\mathrm{H} 14 \mathrm{a}$ & -0.0441 & 0.9656 & 0.3882 & 0.088 \\
\hline$H 14 b$ & -0.0036 & 0.9895 & 0.2734 & 0.088 \\
\hline $\mathrm{H} 14 \mathrm{c}$ & 0.1278 & 0.9960 & 0.4014 & 0.088 \\
\hline C15 & $0.1145(6)$ & $0.7977(4)$ & $0.4821(4)$ & $0.088(2)$ \\
\hline $\mathrm{H} 15 \mathrm{a}$ & 0.0149 & 0.8006 & 0.4957 & 0.131 \\
\hline $\mathrm{H} 15 \mathrm{~b}$ & 0.1960 & 0.8242 & 0.5479 & 0.131 \\
\hline $\mathrm{H} 15 \mathrm{c}$ & 0.1420 & 0.7396 & 0.4764 & 0.131 \\
\hline 01 & $0.6213(3)$ & $0.89120(13)$ & $0.5782(2)$ & $0.0489(6)$ \\
\hline $\mathrm{O} 2$ & $0.6054(2)$ & $0.78179(11)$ & $0.4578(2)$ & $0.0359(5)$ \\
\hline $\mathrm{O} 3$ & $0.5594(2)$ & $0.81295(12)$ & $0.2568(2)$ & $0.0367(5)$ \\
\hline & & & & t \\
\hline
\end{tabular}


Table 3. Refined Thermal Parameters (U's) for Compound 2023

\begin{tabular}{|c|c|c|c|c|c|c|}
\hline Atom & $U_{11}$ & $\mathrm{U}_{22}$ & $\mathrm{U}_{33}$ & $\mathrm{U}_{23}$ & $U_{13}$ & $\mathrm{U}_{12}$ \\
\hline Si1 & $0.0297(5)$ & $0.0465(6)$ & $0.0347(5)$ & $0.0077(3)$ & $0.0117(4)$ & $0.0030(3)$ \\
\hline $\mathrm{C}_{1}$ & $0.0312(13)$ & $0.0288(13)$ & $0.0319(13)$ & $0.0032(10)$ & $0.0112(11)$ & $0.0045(10)$ \\
\hline $\mathrm{C} 2$ & $0.042(2)$ & $0.041(2)$ & $0.055(2)$ & $0.0105(13)$ & $0.0185(14)$ & $0.0005(12)$ \\
\hline C3 & $0.053(2)$ & $0.036(2)$ & $0.053(2)$ & $0.0119(13)$ & $0.022(2)$ & $-0.0019(13)$ \\
\hline $\mathrm{C} 4$ & $0.058(2)$ & $0.039(2)$ & $0.039(2)$ & $0.0096(12)$ & $0.0169(14)$ & $0.0051(13)$ \\
\hline C5 & $0.0367(14)$ & $0.0374(14)$ & $0.0299(13)$ & $0.0075(11)$ & $0.0073(11)$ & $0.0054(11)$ \\
\hline C6 & $0.0312(13)$ & $0.0311(13)$ & $0.0234(12)$ & $-0.0006(10)$ & $0.0069(10)$ & $0.0005(10)$ \\
\hline C7 & $0.0251(12)$ & $0.0301(12)$ & $0.0234(12)$ & $-0.0018(9)$ & $0.0046(10)$ & $-0.0019(9)$ \\
\hline C8 & $0.0269(12)$ & $0.0262(12)$ & $0.0258(12)$ & $-0.0021(9)$ & $0.0037(10)$ & $0.0021(9)$ \\
\hline C9 & $0.0344(13)$ & $0.0274(13)$ & $0.0297(14)$ & $-0.0019(10)$ & $0.0015(11)$ & $0.0005(10)$ \\
\hline C10 & $0.043(2)$ & $0.0291(14)$ & $0.0291(14)$ & $-0.0001(10)$ & $0.0084(12)$ & $0.0046(11)$ \\
\hline $\mathrm{C}+1$ & $0.0390(14)$ & $0.0263(13)$ & $0.0292(13)$ & $-0.0011(10)$ & $0.0085(11)$ & $-0.0017(10)$ \\
\hline C12 & $0.061(2)$ & $0.037(2)$ & $0.050(2)$ & $0.0026(13)$ & $0.020(2)$ & $0.0181(14)$ \\
\hline $\mathrm{C} 13$ & $0.035(2)$ & $0.065(2)$ & $0.071(2)$ & $-0.005(2)$ & $0.014(2)$ & $-0.009(2)$ \\
\hline C14 & $0.048(2)$ & $0.059(2)$ & $0.080(2)$ & $-0.015(2)$ & $0.036(2)$ & $0.001(2)$ \\
\hline C15 & $0.072(3)$ & $0.145(5)$ & $0.060(2)$ & $0.048(3)$ & $0.041(2)$ & $0.034(3)$ \\
\hline 01 & $0.0569(13)$ & $0.0395(12)$ & $0.0307(11)$ & $-0.0047(8)$ & $-0.0099(9)$ & $0.0031(9)$ \\
\hline O2 & $0.0354(10)$ & $0.0318(10)$ & $0.0328(10)$ & $0.0017(7)$ & $0.0019(8)$ & $0.0079(7)$ \\
\hline $\mathrm{O3}$ & $0.0385(10)$ & $0.0352(10)$ & $0.0419(11)$ & $0.0041(8)$ & $0.0208(9)$ & $0.0085(8)$ \\
\hline s. & L & -1 & 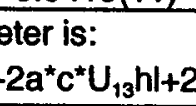 & & & \\
\hline
\end{tabular}


Table 4. Bond Distances in Compound 2023, $\AA$

\begin{tabular}{|llllll|}
\hline Si1-C14 & $1.857(4)$ & Si1-C15 & $1.861(4)$ & Si1-C13 & $1.863(3)$ \\
Si1-C7 & $1.885(3)$ & C1-O3 & $1.449(3)$ & C1-C2 & $1.507(4)$ \\
C1-C6 & $1.535(3)$ & C1-C8 & $1.565(3)$ & C2-C3 & $1.529(4)$ \\
C3-C4 & $1.521(5)$ & C4-C5 & $1.520(4)$ & C5-C6 & $1.524(4)$ \\
C6-C7 & $1.577(3)$ & C7-C11 & $1.549(3)$ & C7-C8 & $1.587(3)$ \\
C8-C9 & $1.491(3)$ & C9-O1 & $1.212(3)$ & C9-O2 & $1.345(3)$ \\
C10-O3 & $1.414(3)$ & C10-O2 & $1.467(3)$ & C10-C12 & $1.508(4)$ \\
C10-C11 & $1.517(4)$ & & & & \\
\hline
\end{tabular}

Table 5. Bond Angles in Compound 2023,

\begin{tabular}{|llllll|}
\hline C14-Si1-C15 & $108.1(2)$ & C14-Si1-C13 & $109.9(2)$ & C15-Si1-C13 & $108.9(2)$ \\
C14-Si1-C7 & $113.16(13)$ & C15-Si1-C7 & $110.9(2)$ & C13-Si1-C7 & $105.74(14)$ \\
O3-C1-C2 & $106.7(2)$ & O3-C1-C6 & $110.0(2)$ & C2-C1-C6 & $119.7(2)$ \\
O3-C1-C8 & $107.1(2)$ & C2-C1-C8 & $123.1(2)$ & C6-C1-C8 & $88.8(2)$ \\
C1-C2-C3 & $112.4(2)$ & C4-C3-C2 & $111.5(3)$ & C5-C4-C3 & $109.3(2)$ \\
C4-C5-C6 & $111.4(2)$ & C5-C6-C1 & $111.7(2)$ & C5-C6-C7 & $116.6(2)$ \\
C1-C6-C7 & $86.9(2)$ & C11-C7-C6 & $105.8(2)$ & C11-C7-C8 & $105.0(2)$ \\
C6-C7-C8 & $86.6(2)$ & C11-C7-Si1 & $112.2(2)$ & C6-C7-Si1 & $120.9(2)$ \\
C8-C7-Si1 & $122.7(2)$ & C9-C8-C1 & $114.7(2)$ & C9-C8-C7 & $116.6(2)$ \\
C1-C8-C7 & $85.6(2)$ & O1-C9-O2 & $119.8(2)$ & O1-C9-C8 & $126.6(2)$ \\
O2-C9-C8 & $113.5(2)$ & O3-C10-O2 & $107.3(2)$ & O3-C10-C12 & $108.2(2)$ \\
O2-C10-C12 & $106.0(2)$ & O3-C10-C11 & $111.5(2)$ & O2-C10-C11 & $106.9(2)$ \\
C12-C10-C11 & $116.3(2)$ & C10-C11-C7 & $108.5(2)$ & C9-O2-C10 & $112.9(2)$ \\
C10-O3-C1 & $111.7(2)$ & & & & \\
\hline
\end{tabular}

